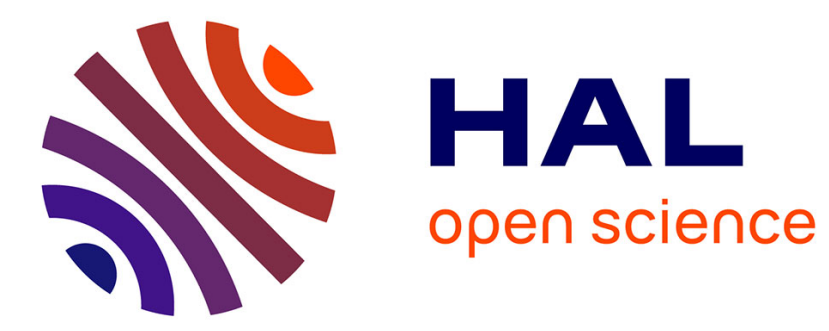

\title{
Sumset Valuations of Graphs and Their Applications
}

Sudev Naduvath, Germina Augustine, Johan Kok

\section{To cite this version:}

Sudev Naduvath, Germina Augustine, Johan Kok. Sumset Valuations of Graphs and Their Applications. Handbook of Research on Advanced Applications of Graph Theory in Modern Society, 2019, 9781522593805. 10.4018/978-1-5225-9380-5.ch009 . hal-02268882

\section{HAL Id: hal-02268882 \\ https://hal.science/hal-02268882}

Submitted on 21 Aug 2019

HAL is a multi-disciplinary open access archive for the deposit and dissemination of scientific research documents, whether they are published or not. The documents may come from teaching and research institutions in France or abroad, or from public or private research centers.
L'archive ouverte pluridisciplinaire HAL, est destinée au dépôt et à la diffusion de documents scientifiques de niveau recherche, publiés ou non, émanant des établissements d'enseignement et de recherche français ou étrangers, des laboratoires publics ou privés. 


\title{
Sumset Valuations of Graphs And Their APPLICATIONS
}

\author{
SudeV NADUVATH* \\ Department of Mathematics, CHRIST (Deemed to be University) \\ Bangalore, Karnataka, INDIA. \\ Germina Augustine \\ Department of Mathematics, Central University of Kerala, \\ Periya, Kasaragod, Kerala, INDIA.

\section{JOHAN KOK} \\ Licensing Services, Metropolitan Police Department \\ City of Tshwane, South Africa.
}

\section{Contents}

1 Introduction $\quad 3$

1.1 Graph Labelling. . . . . . . . . . . . . . . . . . . . . . 3

1.2 Sumsets of Two Sets of Numbers . . . . . . . . . . . . . . . 4

2 Sumset Labelling of Graphs 5

2.1 A Broad Classification of Sumset Graphs . . . . . . . . . . . . . . 6

3 Some Intrinsic Sumset Graphs $\quad 6$

3.1 Weak Sumset Labelling of Graphs . . . . . . . . . . . . . . . . . . . 7

3.2 Strong Sumset Labelling of Graphs . . . . . . . . . . . . . . . . . . . . . . . 9

3.3 Arithmetic Sumset Labelling of Graphs . . . . . . . . . . . . . . . . 10

4 Some Extrinsic Sumset Graphs $\quad \mathbf{1 4}$

4.1 Topological and Topogenic Sumset Graphs . . . . . . . . . . . . . . 14

4.2 Graceful and Sequential Sumset Graphs . . . . . . . . . . . . . . 17

5 Hypergraphs associated with sumset graphs 20

${ }^{*}$ Corresponding author- email:sudevnk@gmail.com (Sudev Naduvath) 
6 Sumset Labelled Signed Graphs $\quad 23$

6.1 Sumset Signed Graphs . . . . . . . . . . . . . . . . . . 23

6.2 Switching of Sumset Signed Graphs . . . . . . . . . . . . . 26

7 Modular Sumset Labelling of Graphs 28

8 Scope for Further Studies $\quad 31$ 


\begin{abstract}
Graph labelling is an assignment of labels to the vertices and/or edges of a graph with respect to certain restrictions and in accordance with certain predefined rules. The sumset of two non-empty sets $A$ and $B$, denoted by $A+B$, is defined by $A+B=\{a+b: a \in A, b \in B\}$. Let $X$ be a nonempty subset of the set $\mathbb{Z}$ and $\mathcal{P}(X)$ be its power set. A sumset labelling of a given graph $G$ is an injective set-valued function $f: V(G) \rightarrow \mathcal{P}_{0}(X)$, which induces a function $f^{+}: E(G) \rightarrow \mathcal{P}_{0}(X)$ defined by $f^{+}(u v)=f(u)+f(v)$, where $f(u)+f(v)$ is the sumset of the set-labels of the vertices $u$ and $v$. This chapter discusses different types of sumset labellings of graphs and their structural characterisations. The properties and characterisations of certain hypergraphs and signed graphs, which are induced by the sumset-labellings of given graphs have also done in this chapter.
\end{abstract}

Keywords: Sumset labelling, weak sumset labelling, strong sumset labelling, topological sumset labelling, graceful sumset labelling, sequential sumset labelling, sumset hypergraphs, sumset signed graphs.

MSC 2010: 05C78, 11B13.

\title{
1 Introduction
}

In graph theory, graph labelling is an assignment of labels to the vertices and/or edges of a graph with respect to certain restrictions and in accordance with certain predefined rules. For the past few decades, graph labelling has become a fruitful research area in graph theory. Different graph labellings have been resulted from practical considerations. They are not only of theoretical interests, but have many practical implications also. For many applications, the edges or vertices of a given graph are given labels that are meaningful in the context.

\section{$1.1 \quad$ Graph Labelling}

A valuation of a graph $G$ is a one to one mapping of the vertex set of $G$ on to the set of all integers $\mathbb{Z}$. Graph labeling has become a fertile research area in graph theory after the introduction of the notion of certain types number valuations, called $\beta$-valuations, of graphs in [54]. The most popular one among the number valuations of graphs is $\beta$-valuation or graceful labelling of a graph (see $[35,54])$ which is defined as an injective function $f: V(G) \rightarrow\{1,2,3, \ldots,|E|\}$ such that, when each edge $x y$ is assigned the label $|f(x)-f(y)|$, the resulting edge labels are distinct. A graph $G(V, E)$ is said to be edge-graceful if there exists a bijection $f$ : $E \rightarrow\{1,2,3, \ldots,|E|\}$ such that the induced mapping $f^{+}: V \rightarrow\{0,1, \cdots,|V|-1\}$ given by $f^{+}(u)=f(u v)(\bmod |V|)$ taken over all edges $u v$ is a bijection.

Motivated by various problems in social networks, a set analogue of number valuations called set-valuation of graphs, has been introduced in [5]. In the number valuations, the elements of a graph are assigned to numbers while in the set-valuations, 
the elements of a graph are assigned to sets subject to certain conditions. The mathematical definitions of a set-valuation of a graph concerned is as given below (see [5]):

For a graph $G$ and a non-empty set $X$, a set-labeling or a set-valuation of $G$, with respect to the set $X$, is an injective set-valued function $f: V(G) \rightarrow \mathcal{P}(X)$ such that the function $f^{\oplus}: E(G) \rightarrow \mathcal{P}(X)-\{\emptyset\}$ is defined by $f^{\oplus}(u v)=f(u) \oplus f(v)$ for every $u v \in E(G)$, where $\mathcal{P}(X)$ is the set of all subsets of $X$ and $\oplus$ is the symmetric difference of sets. The definition can be generalised by allowing any binary operation * of two sets in place of the symmetric difference $\oplus$ in the above definitions.

Many significant and interesting studies on set-labeling of graphs have been done in the last two decades (see [1-10,32,33,91]).

\subsection{Sumsets of Two Sets of Numbers}

The sumset of two non-empty sets $A$ and $B$, denoted by $A+B$, is defined by $A+B=\{a+b: a \in A, b \in B\}$ (c.f. [48]). Note that unlike other operations of sets, sumsets can be defined only for number sets. This area is very much interesting and significant not only because not much articles on sumsets are available in the literature, but also the studies on the structural characteristics and cardinality of sumsets in context of graph labelling are also promising. If either $A$ or $B$ is uncountable, then $A+B$ is also uncountable. Also, $A+\emptyset=\emptyset$. in view these, we consider the finite non-empty subsets of $\mathbb{Z}$, the set of integers for our current discussions.

If $A, B$ and $C$ are non-empty sets of integers such that $C=A+B$, where $A \neq\{0\}$ and $B \neq\{0\}$, then $A$ and $B$ are said to be the non-trivial summands of the set $C$ and $C$ is said to be the non-trivial sumset of $A$ and $B$. It can also be noted that for a non-empty set $A$ containing the element $0, A+\{0\}=A$. In this case $\{0\}$ and $A$ are said to be the trivial summands of $A$. The following result provides the bounds for the cardinality of the sumset of two non-empty sets of integers.

Theorem 1.1 (Nathanson [48]). If $A$ and $B$ are two non-empty sets of integers, then $|A|+|B|-1 \leq|A+B| \leq|A||B|$.

Two ordered pairs $(a, b)$ and $(c, d)$ in $A \times B$ may be compatible if $a+b=c+d$. If $(a, b)$ and $(c, d)$ are compatible, then we write $(a, b) \sim(c, d)$. Note that the compatibility relation $\sim$ is an equivalence relation (see [59,60]). A compatibility class of an ordered pair $(a, b)$ in $A \times B$ with respect to the integer $k=a+b$ is the subset of $A \times B$ defined by $\{(c, d) \in A \times B:(a, b) \sim(c, d)\}$ and is denoted by $[(a, b)]_{k}$ or $C_{k}$. Note that $(a, b) \in[(a, b)]_{k}$, and hence each compatibility class $[(a, b)]_{k}$ is non-empty. If a compatibility class contains exactly one element, then it may be called a trivial compatibility class. The saturation number of a compatibility class, denoted by $\eta\left(\mathrm{C}_{k}\right)$, is the maximum number of elements possible in a compatibility class and a compatibility class with the maximum number of elements is called a saturated compatibility class. It is proved in [60] that the cardinality of a saturated class in $A \times B$ is $\min (|A|,|B|)$.

For example, consider the sets $A=\{2,4,6,8\}$ and $B=\{1,3,5\}$. Then, $A+B=$ 
$\{3,5,7,9,11,13\}$. The compatibility classes in $A \times B$ are

$$
\begin{aligned}
\mathrm{C}_{3} & =\{(2,1)\} \\
\mathrm{C}_{5} & =\{(2,3),(4,1)\} \\
\mathrm{C}_{7} & =\{(2,5),(4,3),(6,1)\} \\
\mathrm{C}_{9} & =\{(4,5),(6,3),(8,1)\} \\
\mathrm{C}_{11} & =\{(6,5),(8,3)\} \\
\mathrm{C}_{13} & =\{(8,5)\} .
\end{aligned}
$$

Here, the compatibility classes $\mathrm{C}_{7}$ and $\mathrm{C}_{9}$ are saturated classes for $A \times B$.

The set $A \times B$ need not always contain a saturated class. In other words, there is a possibility that the cardinality of every compatibility class of $A \times B$ is fewer than $\eta\left(\mathrm{C}_{k}\right)$. In such cases, a compatibility class of $A \times B$ that contains maximum number of elements (more than all other classes of $A \times B$ ), but fewer than $\min (|A|,|B|$ ), is called a maximal compatibility class. Note that all saturated compatibility classes are maximal compatibility classes, but a maximal compatibility class need not be a saturated class, which can be verified from the following example.

Consider the set $A=\{1,2,3\}$ and $B=\{2,3,5\}$. Then, $A+B=\{3,4,5,6,7,8\}$. The compatibility classes are

$$
\begin{aligned}
& \mathrm{C}_{3}=\{(1,2)\} \\
& \mathrm{C}_{4}=\{(1,3),(2,2)\} \\
& \mathrm{C}_{5}=\{(2,3),(3,2)\} \\
& \mathrm{C}_{6}=\{(1,5),(3,3)\} \\
& \mathrm{C}_{7}=\{(2,5)\} \\
& \mathrm{C}_{8}=\{(3,5)\} .
\end{aligned}
$$

In the above example, $C_{4}, C_{5}$ and $C_{6}$ are maximal compatible classes but none of compatibility classes is a saturated class in $A \times B$.

Note that only one representative element of each compatibility class $C_{k}$ of $A \times B$ contributes an element to their sumset $A+B$ and all other $r_{k}-1$ elements are avoided.

\section{Sumset Labelling of Graphs}

Using the concepts of the sumset of two non-empty finite sets of integers, the notion of a sumset labelling of a graph was introduced as follows.

Definition 2.1. [46] Let $X$ be a non-empty subset of the set $\mathbb{Z}$ and $\mathcal{P}(X)$ be its power set. A sumset labelling or an integer additive set-labelling of a given graph $G$ is an injective set-valued function $f: V(G) \rightarrow \mathcal{P}_{0}(X)$, which induces a function $f^{+}: E(G) \rightarrow \mathcal{P}_{0}(X)$ defined by $f^{+}(u v)=f(u)+f(v)$, where $f(u)+f(v)$ is the sumset of the set-labels of the vertices $u$ and $v$. 
It is proved that a sumset labelling can be defined for every graph (see [60]). The graphs with sumset labellings defined on them are called sumset graphs. A sumset indexer or an integer additive set-indexer of a graph $G$ is a sumset labelling $f: V(G) \rightarrow \mathcal{P}_{0}(X)$ of $G$ such that the induced function $f^{+}: E(G) \rightarrow \mathcal{P}_{0}(X)$ defined by $f^{+}(u v)=f(u)+f(v)$ is also injective (see [33]).

As mentioned earlier, unlike other binary operations on sets, the sumset has some unique properties. Hence, the graphs which admits graphs will have many interesting properties including many exciting structural characteristics. This makes the study on sumset graphs significant and promising.

\subsection{A Broad Classification of Sumset Graphs}

The sumset labellings of graphs (and hence the sumset graphs) can be broadly classified into two categories.

(a) Intrinsic Sumset Graphs: This type includes the sumset graphs which are characterised with respect to the nature of the (individual) set-labels and their elements. The Type-I sumset graphs are classified in terms of the nature of elements in the set-labels (such as arithmetic progressions, sequences etc.) and the cardinality of the set-labels.

(b) Extrinsic Sumset Graphs: This type includes the sumset graphs which are characterised in terms of the nature of the collection of the set-labels of the graphs. That is, the graphs are classified according to whether the collection of vertex set-labels or the collection of the edge set-labels or both together follow certain properties. For example, the characterisation of sumset graphs is done in accordance with whether the collection of set-labels forms a topology of the ground set, the power set of the ground set, a filter of the ground set etc.

In the following discussion, we will investigate the properties and structural characterisations of the graphs which admit the above-mentioned types of sumset graphs.

\section{Some Intrinsic Sumset Graphs}

The cardinality of the set-label of an element (vertex or edge) of a graph $G$ is called the set-indexing number of that element. A sumset labelling is said to be $k$-uniform if $\left|f^{+}(e)\right|=k, \forall e \in E(G)$. In other words, a connected graph $G$ is said to have a $k$-uniform sumset labelling if all of its edges have the same setindexing number $k$. The vertex set $V(G)$ is said to be l-uniformly set-indexed, if $|f(v)|=l, \forall v \in V(G)$. A graph is said to be $(k, l)$-uniform if $|f(v)|=l, \forall v \in V(G)$ and $\left|f^{+}(e)\right|=k, \forall e \in E(G)$.

Let $X$ be a finite set of integers and let $f: V(G) \rightarrow \mathcal{P}_{0}(X)$ be a sumset labelling of a graph $G$ on $n$ vertices. Then, it can be observed that $X$ contains at least $\left\lceil\log _{2}(n+1)\right\rceil$ elements (see $[46,60]$. It can be observed that if $V(G)$ is $l$-uniformly set-indexed, then the cardinality of $X$ is given by $\left(\begin{array}{c}|X| \\ l\end{array}\right) \geq n(c f .[46,60])$. 
Three types of sumset labellings of graphs, based on the cardinalities of the setlabels assigned to the elements of graphs, have broadly been studied in the recent literature. They are: strong, weak and arithmetic sumset-labellings. If $f$ is a sumset labelling of $G$, then by Theorem 1.1, it can be noted that

$$
|f(u)|+|f(v)|-1 \leq\left|f^{+}(u v)\right|=|f(u)+f(v)| \leq|f(u)||f(v)| .
$$

The notion of weak sumset labelling originated from the question whether the set-indexing numbers of edges could be equal to those of their end vertices. By the definition of the sumsets, we know that $|A|,|B| \leq|A+B|$. What are the conditions required for the existence of equality in this condition? By Theorem 1.1, one can observe that $|A+B|=|A|$ if and only if $|B|=1$. Hence, the cardinality of the sumset of two sets is equal to the cardinality of one of them if and only if the other set is a singleton set. Moreover, $|A+B|=|A|=|B|$ if and only if the sets $A$ and $B$ and the sumset $A+B$ are all singleton sets. The elements (vertices and edges) with singleton set-labels (that is, with set-indexing number 1) may be called mono-indexed elements. The investigations on the lower bound in the above expression, lead us to the notions of different types of arithmetic sumset labellings and the investigations on the upper bound provided the concepts and results of strong sumset labelling of graphs. We shall now go through all these types of sumset labellings one by one.

\subsection{Weak Sumset Labelling of Graphs}

Definition 3.1. Let $f: V(G) \rightarrow \mathcal{P}_{0}(X)$ be a sumset labelling defined on a graph $G$. Then, $f$ is said to be a weak sumset labelling if the set-indexing number of every edge of $G$ is equal to the set-indexing number of one or both of its end vertices.

A graph with a weak sumset labelling defined on it may be called a weak sumset graph. Invoking the properties of the cardinalities of non-empty sets and their sumsets mentioned above, a necessary and sufficient condition for a graph $G$ to have a weak sumset labelling has been established in $[60,61]$ as follows.

Lemma 3.1. A sumset labelling $f$ of a graph $G$ is a weak sumset labelling of $G$ if and only if at least one end vertex of every edge in $G$ has a singleton set-label.

In other words, a graph $G$ is a weak sumset graph if and only if it is bipartite or it has mono-indexed edges. The minimum number of mono-indexed edges required for a graph $G$ to admit a weak sumset labelling is said to be the sparing number of $G$. The sparing number of a graph $G$ is denoted by $\varphi(G)$. For any subgraph $H$ of a sumset labelling-graph $G$, it can be noted that the induced mapping $\left.f\right|_{H}$ is a weak sumset labelling for $H$. Therefore, for any subgraph $H$ of a sumset labelling-graph $G, \varphi(H) \leq \varphi(G)$.

A set of vertices $V^{\prime}$ of a graph $G$ is said to have the maximal incidence in $G$, if the number of edges in $G$ having at least one end vertex in $V^{\prime}$ is maximum over all other set of vertices in $G$. The following results discuss the sparing number of arbitrary graphs. 
Theorem 3.2. Let $G$ be a given weak sumset graph and $I$ be an independent set in $G$ which has the maximal incidence in $G$. Then, the sparing number of $G$ is the $|E(G-I)|$.

Theorem 3.3. Let $G(V, E)$ be a connected weak sumset labelling-graph and let $E_{I}$ be a maximal set of edges of $G$ whose one end vertex is in an independent set of $G$. Then, the sparing number of $G$ is $|E|-\left|E_{I}\right|$.

Theorem 3.4. If $G(V, E)$ be a non-empty graph and $I^{*}$ be an independent set having maximum incidence in $G$. Then $\varphi(G)=|E|-\sum_{v_{i} \in I^{*}} d\left(v_{i}\right)$.

The sparing number of an $r$-regular graph is determined in the following result.

Corollary 3.5. If $G$ is an $r$-regular graph, then $\varphi(G)=|E|-r \alpha$, where $\alpha$ is the independence number of $G$.

The spring number of different graph classes, derived graph classes, graph operations and graph products have been discussed in recent literature (ref [23-25, 6169,71]). The sparing numbers of different graph classes are given in Table .

The following result discussed the sparing number of the union of two graphs.

Theorem 3.6. Let $G_{1}$ and $G_{2}$ be two subgraphs of a graph $G$ such that $G=G_{1} \cup G 2$. Then, $\varphi(G)=\varphi\left(G_{1}\right)+\varphi\left(G_{2}\right)-\varphi\left(G_{1} \cap G_{2}\right)$. Moreover, if $G_{1}$ and $G_{2}$ are edge-disjoint subgraphs of $G$, then $\varphi\left(G_{1} \cup G_{2}\right)=\varphi\left(G_{1}\right)+\varphi\left(G_{2}\right)$.

The following results discussed the sparing number of the join of two graphs.

Theorem 3.7. The sparing number of the join of two non-empty graphs $G_{1}\left(V_{1}, E_{1}\right)$ and $G_{2}\left(V_{2}, E_{2}\right)$ is $\min \left\{\varphi\left(G_{1}\right)+\left|E_{2}\right|\left(\beta_{1}+1\right), \varphi\left(G_{2}\right)+\left|E_{1}\right|\left(\beta_{2}+1\right)\right\}$, where $\beta_{1}, \beta_{2}$ are the covering numbers of $G_{1}$ and $G_{2}$ respectively.

Theorem 3.8. Let $G$ be the join of two graphs $G_{1}\left(V_{1}, E_{1}\right)$ and $G_{2}\left(V_{2}, E_{2}\right)$. Then, $\varphi\left(G_{1}+G_{2}\right)=\left|E_{1}\right|+\left|E_{2}\right|+\left|V_{1}\right|\left|V_{2}\right|-\max \left\{\left(\left|V_{2}\right|\left(\left|I_{1}\right|-1\right)+\left|E_{I_{1}}\right|\right),\left(\left|V_{1}\right|\left(\left|I_{2}\right|-1\right)+\left|E_{I_{2}}\right|\right)\right\}$, where, for $j=1,2$ and $I_{j}$ is an independent set of the graph $G_{j}$ with maximal incidence in $G_{j}$ and $E_{I_{j}}$ is the set of edges in $G_{1}+G_{2}$ with their one end vertex in $I_{j}$.

The sparing number of the Cartesian product two weak sumset graphs is determined in the following result (see $[46,69])$.

Theorem 3.9. Let $G_{1}\left(V_{1}, E_{1}\right)$ and $G_{2}\left(V_{2}, E_{2}\right)$ be two non-empty graphs. Then, the sparing number of their Cartesian product $G_{1} \square G_{2}$ is

$$
\varphi\left(G_{1} \square G_{2}\right)=\left|V_{1}\right| \varphi\left(G_{2}\right)+\left|V_{2}\right| \varphi\left(G_{1}\right) .
$$

The sparing number of the Corona product two weak sumset graphs is determined in the following result (see [46, 69]).

Theorem 3.10. Let $G_{1}\left(V_{1}, E_{1}\right)$ and $G_{2}\left(V_{2}, E_{2}\right)$ be two non-empty graphs. The sparing number of their corona is given by $\varphi\left(G_{1} \odot G_{2}\right)=\varphi\left(G_{1}\right)+\left|V_{1}\right|\left|E_{2}\right|$. 
The set-labelling number of a given graph $\mathrm{G}$ is the minimum cardinality of the ground set $X$ so that the function $f: V(G) \rightarrow \mathcal{P}_{0}(X)$ is a sumset labelling of $G$. Then, set-labelling number of a graph $\mathrm{G}$ is denoted by $\varsigma(G)$. Then, the weak set-labelling number of a graph $G$ is the minimum cardinality of the ground set $X$, such that $f$ is a weak sumset labelling of $G$ and is denoted by $\varsigma *(G)$. The weak set-labelling number of some fundamental graphs have been determined in [70] (see Table ).

For $k>1$, graph $G$ admits a weakly $k$-uniform sumset labelling, if and only if one end vertex of every edge of $G$ is mono-indexed and the other end vertex must be set-labelled by distinct non-singleton sets of cardinality $k$. That is, a graph $G$ is a weakly uniform sumset graph if and only if it is bipartite. Moreover, note that the sparing number of bipartite graphs is 0 .

\subsection{Strong Sumset Labelling of Graphs}

Definition 3.2. Let $f$ is a sumset labelling defined on a given graph $G$. Then $f$ is said to be a strong sumset labelling of $G$ if $\left|f^{+}(u v)\right|=|f(u)+f(v)|=|f(u)||f(v)|$.

A graph which admits a strong sumset labelling or a strong sumset indexer is called a strong sumset graph or a strong sumset graph. If $f$ is a strong sumset labelling of a graph $G$, then it can be noted that the compatibility classes in the Cartesian product of the set-labels of any two adjacent vertices of $G$ are all trivial classes.

The difference-set of a set $A$, denoted by $D_{A}$ is the set defined by $D_{A}=\{|a-b|$ : $a, b \in A\}$. In view of this notion, the following theorem established a necessary and sufficient condition for the sumset labelling $f$ of a graph $G$ to be a strong sumset labelling of $G$.

Theorem 3.11. [46,73] A graph $G$ admits a strong sumset labelling if and only if the difference-sets of the set-labels of any two adjacent vertices in $G$ are disjoint.

The minimum number of pairwise-disjoint difference-sets of the set-labels of vertices required in a graph $G$ so that $G$ admits a strong sumset labelling is called the nourishing number of $G$ and is denoted by $\varkappa(G)$. Any two vertices in a complete graph $K_{n}$ are adjacent to each other and hence for $K_{n}$ to admit a strong sumset labelling, the difference-sets of the set-labels of all its vertices must be pairwise disjoint. Therefore, the nourishing number of $K_{n}$ is $n$. Therefore, for any graph on $n$ vertices, we have $\varkappa(G) \leq n$. Furthermore, if $G$ contains a complete subgraph $H$ of order $r \leq n$, then the set-labels of all vertices of $H$ must have pairwise disjoint difference-sets. The nourishing numbers of certain fundamental graph classes and graph powers have been determined in [74]. (see Table ).

If a graph $G$ admits a strongly $k$-uniform sumset labelling, then it can be noted that the set-indexing number of every edge of $G$ is the product of the set-indexing numbers of its end vertices. Hence, if $G$ is a strongly $k$-uniform sumset graph, then each vertex of $G$ must have some set-indexing number $d_{i}$, which is a divisor of $k$. Hence, $V(G)$ can be partitioned into at most $n$ sets, say $\left(X_{1}, X_{2}, \ldots, X_{l}\right)$ such that each $X_{i}$ consists of the vertices of $G$ having the set-indexing number $d_{i}$, where $l$ is 
the number of divisors of the integer $k$. In [60] it is established that all bipartite graphs admit a strongly $k$-uniform sumset labelling, for any positive integer $k$.

The following theorem established a condition required for a complete graph to admit a strongly $k$-uniform sumset labelling.

Theorem 3.12. [73] A strongly $k$ - uniform sumset labelling of a complete graph $K_{n}$ is a $(k, l)$-uniform sumset labelling, where $l=\sqrt{k}$.

In view of the fact that the number of divisors of a non-square integer is even and the number of divisors of a perfect square integer is odd, we have an interesting results as stated below:

Theorem 3.13. [73] Let $k$ be a non-square integer. Then, a graph $G$ admits a strongly $k$-uniform sumset labelling if and only if $G$ is bipartite or a union of disjoint bipartite components.

Theorem 3.14. [73] Let the graph $G$ has a strongly $k$-uniform sumset labelling. Then

(i) G contains at most one component that is a clique.

(ii) If $G$ has a component which is a clique, then $k$ is a perfect square.

As a result of the above theorems, one can infer that a connected non-bipartite graph $G$ admits a strongly $k$-uniform sumset labelling if and only if $k$ is a perfect square and this sumset labelling is $(k, l)$-uniform, where $l=\sqrt{k}$.

\subsection{Arithmetic Sumset Labelling of Graphs}

In [48], it is proved that $|A+B|=|A|+|B|-1$ if and only if the elements of both $A$ and $B$ are in arithmetic progressions with same common difference. Hence, in this context, the graphs whose vertices and edges having the set-labels are arithmetic progressions arise much interest. If context is clear, the common difference of the set-label of an element of a graph may be called the common difference of that element. The deterministic ratio of an edge of $G$ is the ratio, $k \geq 1$ between the deterministic indices of its end vertices. It is also assumed that every set-label of the elements of $G$ should have at least three elements.

Definition 3.3. An arithmetic sumset labelling of a graph $G$ is a sumset labelling $f$ of $G$, with respect to which the set-labels of all vertices and edges of $G$ are arithmetic progressions. Similarly, an arithmetic sumset indexer of $G$ is a sumset labelling of $G$, such that $f^{+}$is also injective.

A graph with an arithmetic sumset labelling is called an arithmetic sumset graph. The following result provides a necessary and sufficient condition for a graph to admit an arithmetic sumset labelling.

Theorem 3.15. A graph $G$ admits an arithmetic sumset labelling $f$ if and only if for any two adjacent vertices in $G$, the deterministic ratio of every edge of $G$ is a positive integer, which is less than or equal to the set-indexing number of its end vertex having smaller common difference. 
The set-indexing number of an edge of an arithmetic sumset graph $G$, in terms of the set-indexing numbers of its end vertices, is determined in the following theorem.

Theorem 3.16. Let $G$ be a arithmetic sumset graph and let $d_{i}$ and $d_{j}$ be the common differences of two adjacent vertices $v_{i}$ and $v_{j}$ in $G$. If $\left|f\left(v_{i}\right)\right| \leq\left|f\left(v_{j}\right)\right|$, then for some positive integer $1 \leq k \leq\left|f\left(v_{i}\right)\right|$, the edge $v_{i} v_{j}$ has the set-indexing number $\left|f\left(v_{i}\right)\right|+k\left(\left|f\left(v_{j}\right)\right|-1\right)$, where $f$ is an arithmetic sumset labelling of $G$.

We note that if the set-labels of two adjacent vertices are arithmetic progressions with the same common difference, say $d$, then the set-label of the corresponding edge is also an arithmetic progression with same common difference $d$. Hence, we have

Definition 3.4. $[76,78]$ Let $f$ be an arithmetic sumset labelling defined on a given graph $G$. If all the elements of $G$ have the same common difference under $f$, then $f$ is said to be an isoarithmetic sumset labelling.

A graph which has an isoarithmetic sumset labelling is called an isoarithmetic sumset graph. It is proved that every graph $G$ can have an isoarithmetic sumset labelling ( $c f .[76])$. Invoking Theorem 1.1, the set-indexing number of edges of an isoarithmetic sumset graph can be found out in [76] as stated in the following result.

Theorem 3.17. Let $G$ be a graph with an arithmetic sumset labelling $f$ defined on it. Then, $f$ is an isoarithmetic sumset labelling on $G$ if and only if the set-indexing number of every edge of $G$ is one less than the sum of the set-indexing numbers of its end vertices.

The following theorems answer the questions whether an isoarithmetic sumset labelling $f$ defined on a graph $G$ be a uniform sumset labelling.

Theorem 3.18. An isoarithmetic sumset labelling of a graph $G$ is a uniform sumset labelling if and only if $V(G)$ is uniformly set-indexed or $G$ is bipartite.

Theorem 3.19. An isoarithmetic sumset labelling $f$ of a graph $G$ is an $r$-uniform sumset labelling if and only if every component of $G$ is either bipartite or its vertex set is l-uniformly set-indexed, where $l=\frac{1}{2}(r+1)$.

Let $f$ be an arithmetic sumset labelling defined on a given graph $G$ such that $V(G)$ is $l$-uniformly set-indexed. Then, in view of the above theorem, one can observe that $f$ is an isoarithmetic sumset labelling of $G$ if and only if $G$ is a $(2 l-1)$ uniform sumset graph (see [77]. It is also proved in [78] that no isoarithmetic sumset labelling defined on a given graph $G$ can be a strong sumset labelling of $G$. This result immediate from the fact that $m+n-1=m n$ if and only if either $m=1$ or $n=1$.

For an isoarithmetic sumset graph $G$, the number of saturated classes in the Cartesian product of the set-labels of any two adjacent vertices in $G$ is one greater than the difference between cardinality of the set-labels of these vertices. Moreover, exactly two compatibility classes, other than the saturated classes, have the same cardinality in the Cartesian product of the set-labels of these vertices (see [78]. Also, if $V(G)$ is $l$-uniformly set-indexed, then there exists exactly one saturated class in the Cartesian product of the set-labels of any two adjacent vertices in $G$ (see [78]. 
Definition 3.5. An arithmetic sumset labelling $f$ of a graph $G$, under which the deterministic ratio of each edge $e$ of $G$ is a positive integer greater than 1 and less than or equal to the set-indexing number of the end vertex of $e$ having smaller common is called a biarithmetic sumset labelling of $G$. If the deterministic ratio of every edge of $G$ is the same, then $f$ is called an identical biarithmetic sumset labelling.

In other words, a biarithmetic sumset labelling of a graph $G$ is an arithmetic sumset labelling $f$ of $G$, for which the common differences of any two adjacent vertices $v_{i}$ and $v_{j}$ in $G$, denoted by $d_{i}$ and $d_{j}$ respectively such that $d_{i}<d_{j}$, holds the condition $d_{j}=k d_{i}$ where $k$ is a positive integer such that $1<k \leq\left|f\left(v_{i}\right)\right|$. A graph $G$ with a biarithmetic sumset labelling may be called a biarithmetic sumset graph.

The following theorem states a necessary and sufficient condition for a graph to have an identical biarithmetic sumset labelling.

Theorem 3.20. [76] A graph $G$ admits an identical biarithmetic sumset labelling if and only if it is bipartite.

The following theorem describes the set-indexing number of the edges of a biarithmetic sumset graph.

Theorem 3.21. [76] Let $G$ be a graph which admits an arithmetic sumset indexer, say $f$. Let $v_{i}$ and $v_{j}$ be two adjacent vertices in $G$ with the deterministic indices $d_{i}$ and $d_{j}$, such that $d_{j}=k d_{i}$, where $k$ is a positive integer such that $1<k \leq\left|f\left(v_{i}\right)\right|$. Then, the set-indexing number of the edge $v_{i} v_{j}$ is $\left|f\left(v_{i}\right)\right|+k\left(\left|f\left(v_{j}\right)\right|-1\right)$.

A necessary and sufficient condition for a biarithmetic sumset labelling of $G$ to be a strong sumset labelling is described in the result given below.

Theorem 3.22. [76] A biarithmetic sumset labelling $f$ of a graph $G$ is a strong sumset labelling of $G$ if and only if the deterministic ratio of every edge of $G$ is equal to the set-indexing number of its end vertex having smaller deterministic index.

Invoking Theorem 3.22, it is proved that an identical biarithmetic sumset labelling of a graph $G$ is a strong sumset labelling of $G$ if and only if one partition of $V(G)$ is $k$-uniformly set-indexed, where $k$ is the deterministic ratio of the edges of $G$.

Let $G$ be a biarithmetic sumset graph. Let $v_{i}$ and $v_{j}$ be two adjacent vertices in $G$, where $v_{i}$ has the smaller common difference. Let $k$ be the deterministic ratio of the edge $v_{i} v_{j}$. Then, a compatible class in $f\left(v_{i}\right) \times f\left(v_{j}\right)$ is a saturated class if and only if $\left|f\left(v_{i}\right)\right|=\left(\left|f\left(v_{j}\right)\right|-1\right) k+r, r>0$. Also, the number of saturated classes in $f\left(v_{i}\right) \times f\left(v_{j}\right)$ is $\left|f\left(v_{i}\right)\right|-\left(\left|f\left(v_{j}\right)\right|-1\right) k$ (see [78]. Moreover, for $1 \leq p \leq n-1$, there are exactly $2 k$ compatibility classes containing $p$ elements. It is clear that if $f\left(v_{i}\right)<f\left(v_{j}\right)$, then there is no saturated class in $f\left(v_{i}\right) \times f\left(v_{j}\right)$. Moreover, if $\left|f\left(v_{i}\right)\right|=p k+q$, where $p, q$ are integers such that $p \leq\left(\left|f\left(v_{j}\right)\right|-1\right)$ and $q<k$, then

(i) if $q=0$, then $\left(\left|f\left(v_{j}\right)\right|-p+1\right) k$ compatibility classes are maximal compatibility classes and contain $p$ elements. 
(ii) if $q>0$, then $\left(\left|f\left(v_{j}\right)\right|-p-1\right) k+q$ compatibility classes are maximal compatibility classes and contain $(p+1)$ elements.

Definition 3.6. [79] A prime arithmetic sumset labelling of a graph $G$ is an arithmetic sumset labelling $f: V(G) \rightarrow \mathcal{P}_{0}(X)$, where for any two adjacent vertices in $G$ the deterministic index of one vertex is a prime multiple of the common difference of the other, where this prime integer is less than or equal to the set-indexing number of the second vertex.

In other words, an arithmetic sumset labelling $f$ is a prime arithmetic sumset labelling of $G$ if for any two adjacent vertices $v_{i}$ and $v_{j}$ of $G$ with the common differences $d_{i}$ and $d_{j}$ respectively, such that $d_{i} \leq d_{j}, d_{j}=p_{i} d_{i}$ where $p_{i}$ is a prime number such that $1<p_{i} \leq\left|f\left(v_{i}\right)\right|$ (refer to [79]. The following theorem states a necessary and sufficient condition for a graph to admit a prime arithmetic sumset labelling.

Theorem 3.23. [79] A graph $G$ admits a prime arithmetic sumset labelling if and only if it is bipartite.

A vertex arithmetic sumset labelling (or edge arithmetic sumset labelling) of a graph is a sumset labelling of $G$ in which all vertex set-labels (or edge set-labels) are arithmetic progressions. If all the vertex set-labels of a graph $G$ are arithmetic progressions and the edge set-labels are not arithmetic progressions with respect to a sumset-labelling, then such a sumset labelling is called semi-arithmetic sumset labelling ( $f f$. [80]).

A vertex-arithmetic sumset labelling $f$ of a graph $G$, under which the differences $d_{i}$ and $d_{j}$ of the set-labels $f\left(v_{i}\right)$ and $f\left(v_{j}\right)$ respectively for two adjacent vertices $v_{i}$ and $v_{j}$ of $G$, holds the conditions $d_{j}=k d_{i}$ and $k$ is a non-negative integer greater than $\left|f\left(v_{i}\right)\right|$ is called the semi-arithmetic sumset labelling of the first kind (cf. [80]). A vertex-arithmetic sumset labelling $f$ of a graph $G$, under which the differences $d_{i}$ and $d_{j}$ of the set-labels $f\left(v_{i}\right)$ and $f\left(v_{j}\right)$ respectively for two adjacent vertices $v_{i}$ and $v_{j}$ of $G$ are not multiples of each other, is called the semi-arithmetic sumset labelling of the second kind ( $c f$. [80]).

It is proved in [80] that every first kind semi-arithmetic sumset labelling of a graph $G$ is a strong sumset labelling of $G$. The following result established a necessary and sufficient condition for a semi-arithmetic sumset labelling of $G$ to be a uniform sumset labelling.

Theorem 3.24. [80] A semi-arithmetic sumset labelling of first kind of a graph $G$ is a uniform sumset labelling if and only if either $G$ is bipartite or $V(G)$ is uniformly set-indexed.

The following theorem discusses the conditions required for a semi-arithmetic sumset labelling of the second kind to be a strong sumset labelling.

Theorem 3.25. [80] Let $f$ be an semi-arithmetic sumset labelling defined on $G$. Also, let $\left|f\left(v_{j}\right)\right|=q .\left|f\left(v_{i}\right)\right|+r, 0<r<\left|f\left(v_{i}\right)\right|$. Then, $f$ is a strong sumset labelling if and only if $q>\left|f\left(v_{i}\right)\right|$ or the differencesd ${ }_{i}$ and $d_{j}$ of two set labels $f\left(v_{i}\right)$ and $f\left(v_{j}\right)$ respectively, are relatively prime. 
One can note that an arithmetic sumset labelling with arbitrary differences need not have any saturated compatibility classes. Let $f$ be an arithmetic sumset labelling with arbitrary common differences on a graph $G$ and $\left|f\left(v_{j}\right)\right|=q \cdot\left|f\left(v_{i}\right)\right|+r$. Also, let $q_{1}$ and $q_{2}$ be the positive integers such that $q_{1} \cdot \mid f\left(v_{j}\right)=q_{2} \cdot r$. Then, the number of elements in a maximal compatible class of $f\left(v_{i}\right) \times f\left(v_{j}\right)$ is $\left\lfloor\frac{\left|f\left(v_{j}\right)\right|}{q_{1}}\right\rfloor$.

Let $f$ be a semi-arithmetic sumset labelling of first kind of a graph $G$ and let $v_{i}$ and $v_{j}$ be two adjacent vertices in $G$. Then, we can observe that all the compatibility classes in $f\left(v_{i}\right) \times f\left(v_{j}\right)$ are trivial classes.

\section{Some Extrinsic Sumset Graphs}

Another classification of sumset graphs is based on the nature and structure of the collection of set-labels of the vertices and edges of graphs. In this section, we examine different types of sumset labellings in this category.

\subsection{Topological and Topogenic Sumset Graphs}

Definition 4.1. Let $G$ be a graph and let $X$ be a non-empty set of integers. A sumset labelling $f: V(G) \rightarrow \mathcal{P}_{0}(X)$ is called a topological sumset labelling of $G$ if $f(V(G)) \cup\{\emptyset\}$ is a topology on $X$. A graph $G$ which admits a topological sumset labelling is called a topological sumset graph.

For a finite set $X$ of integers, let the given function $f: V(G) \rightarrow \mathcal{P}_{0}(X)$ be a sumset labelling on a graph $G$. Since the set-label of every edge $u v$ is the sumset of the sets $f(u)$ and $f(v)$, it can be observed that $\{0\}$ cannot be the set-label of any edge of $G$. Moreover, since $f$ is a topological sumset labelling defined on $G, X$ must be the set-label of some vertex, say $u$, of $G$ and hence the set $\{0\}$ will be the set-label of a vertex, say $v$, and the vertices $u$ and $v$ are adjacent in $G$.

Let $f$ be a topological sumset labelling of a given graph $G$ with respect to a non-empty finite ground set $X$. Then, $\mathcal{T}=f(V(G)) \cup\{\emptyset\}$ is a topology on $X$. Then, the graph $G$ is said to be a $f$-graphical realisation (or simply $f$-realisation) of $\mathcal{T}$. The elements of the sets $f(V)$ are called $f$-open sets in $G$. Existence of graphical realisations for certain topologies of a given set $X$ is established in the following theorem.

Theorem 4.1. [77] Let $X$ be a non-empty finite set of integers. A topology $\mathcal{T}$ of $X$, consisting of the set $\{0\}$ is graphically realisable.

Let $X$ be the ground set and $\mathcal{T}$ be a topology on $X$ and let $f: V(G) \rightarrow \mathcal{P}_{0}(X)$ is a topological sumset labelling of a graph $G$. One can observe following structural properties of a topological sumset graph.

(i) An element $x_{r}$ in $X$ can be an element of the set-label $f(v)$ of a vertex $v$ of $G$ if and only if $x_{r}+x_{s} \leq l$, where $x_{s}$ is any element of the set-label of an adjacent vertex $u$ of $v$ in $G$ and $l$ is the maximal element in $X$. 
(ii) The vertices whose set-labels containing the maximal element of the ground set $X$ are pendant vertices which are adjacent to the vertex having the setlabel $\{0\}$.

(iii) If $G$ has only one pendant vertex and if $G$ admits a topological sumset labelling, then $X$ is the only vertex set-label in $G$, which contains the maximal element of $X$.

(iv) the minimum number of pendant edges incident on a particular vertex of a topological sumset graph is equal to the number of $f$-open sets in $f(V(G))$ containing the maximal element of the ground set $X$,

(v) the minimum number of pendant vertices of a topological sumset graph $G$ is the number of $f$-open sets in $\mathcal{T}$, each of which is the non-trivial summand of at most one $f$-open set in $\mathcal{T}$.

A necessary and sufficient condition for a given graph to admit a topological sumset labelling is provided in the following theorem.

Theorem 4.2. [77] A graph $G$ admits a topological sumset labelling if and only if $G$ has at least one pendant vertex.

In view of Theorem 4.2, it is to be noted that all path graphs and trees admit topological sumset labellings. For $n \geq 3$, neither a cycle $C_{n}$ nor a complete graph $K_{n}$ admits a topological sumset labelling. For $k \geq 2$, no $k$-connected graph admits a topological sumset labelling. Also, for $m, n \geq 2$, no complete bipartite graph $K_{m, n}$ admits a topological sumset labelling.

Let $G$ be a graph with a pendant vertex $v$ which admits a topological sumset labelling, say $f$, with respect to a ground set $X$. Let $f_{1}$ be the restriction of $f$ to the graph $G-v$. Then, there exists a collection $\mathcal{B}$ of proper subsets of $X$ which, together with $\{\emptyset\}$, form a topology on the union of all elements of $\mathcal{B}$ (see [77]).

If $\mathcal{T}$ is the indiscrete topology of the ground set $X$, the only graph $G$ which admits a topological sumset labelling with respect $\mathcal{T}$ is the trivial graph $K_{1}$. If $X$ is a two point set, say $X=\{0,1\}$, then the topology $\mathcal{T}_{1}=\{\emptyset,\{0\}, X\}$ and $\mathcal{T}_{2}=\{\emptyset,\{1\}, X\}$ are the Sierpinski's topologies. Then, the only graph $G$ which admits a topological sumset labelling with respect to a Sierpinski's topology is the graph $K_{2}$. A graph $G$, on $n$ vertices, admits a topological sumset labelling with respect to the discrete topology on the ground set $X$ if and only if $G$ has at least $2^{|X|-1}$ pendant vertices which are adjacent to a single vertex of $G$ (see [77]). In view of this fact, we observe that a graph on even number of vertices does not admit a topological sumset labelling with respect to the discrete topology on the ground set $X$. A star graph $K_{1, r}$ admits a topological sumset labelling with respect to the discrete topology on the ground set $X$, if and only if $r=2^{|X|}-2$.

Definition 4.2. [84] Let $X$ be a finite non-empty set of integers. A sumset labelling $f$ of a given graph $G$, defined by $f: V(G) \rightarrow \mathcal{P}_{0}(X)$, is said to be a topogenic sumset labelling of $G$, with respect to the ground set $X$, if $\mathcal{T}=f(V(G)) \cup f^{+}(E(G)) \cup\{\emptyset\}$ is a topology on $X$. 
A graph $G$ that admits a topogenic sumset labelling is said to be a topogenic sumset graph. Let $\mathcal{T}$ be a topology on $X$, which is neither the indiscrete topology nor the discrete topology on $X$. Then, $\mathcal{T}_{1}=\{\emptyset,\{a\}, X\}$ where $a \in X$. Then, the graph $K_{2}$ admits a topogenic sumset labelling with respect to the set $X$ if and only if $a=0$.

If $f: V(G) \rightarrow \mathcal{P}_{0}(X)$ is a topogenic sumset labelling of $G$, then it is possible for a vertex $v \in V(G)$ and an edge $e \in E(G)$, that $f(v)=f^{+}(e)$. In the following lemma, we discuss the minimum number of elements in a topogenic sumset graph $G$ having the same set-labels.

Let $X$ be a non-empty finite ground set and let $\mathcal{T}$ be a topology on $X$. Then, a graph $G$ is said to be a topogenic graphical realisation of $\mathcal{T}$ if there exists a topogenic sumset labelling $f$ on $G$ such that $f(V(G)) \cup f^{+}(E(G)) \cup\{\emptyset\}=\mathcal{T}$. The result below described a necessary and sufficient condition for the existence of a topogenic graphical realisation for a given topology on the ground set $X$.

Theorem 4.3. [84] Let $X$ be a non-empty finite ground set and let $\mathcal{T}$ be a topology on $X$. Then, a graph $G$ is a topogenic graphical realisation of $\mathcal{T}$ if and only if every non-empty set of $\mathcal{T}$ is either a summand of some other elements of $\mathcal{T}$ or the sumset of two elements of $\mathcal{T}$.

The following theorem determined the order and size of a topogenic sumset graph.

Theorem 4.4. Let $G$ be a topogenic sumset graph with respect to a topology $\mathcal{T}$ of a given ground set $X$ and let $\mathcal{T}$ contains $\rho$ sets which are not the sumsets of any other sets in $\mathcal{T}$. Then, $|V(G)| \geq \rho$ and $|E(G)| \geq \tau-\rho$, where $\tau=|\mathcal{T}|$.

Given a set $X$, a partial ordering $\subseteq$ can be defined on the power set $\mathcal{P}(X)$ by subset inclusion, turning $(\mathcal{P}(X), \subseteq)$ into a lattice. A filter on $X$, denoted by $\mathcal{F}$, is a non-empty subset of the power set $\mathcal{P}(X)$ of $X$ which has the following properties.

(i) $\emptyset \notin \mathcal{F}, X \in \mathcal{F}$.

(ii) $A, B \in \mathcal{F} \Longrightarrow A \cap B \in \mathcal{F}$.

(iii) $A \in \mathcal{F}, A \subset B, \Longrightarrow B \in \mathcal{F}$ where $B$ is a non-empty subset of $X$.

In view of Definition of filters mentioned above, the notion of a sumset filter labelling of a graph is defined as follows.

Definition 4.3. [85] Let $X$ be a finite set of integers. Then, a sumset labelling $f: V(G) \rightarrow \mathcal{P}_{0}(X)$ is said to be a sumset filter labelling of $G$ if $\mathcal{F}=f(V)$ is a proper filter on $X$. A graph $G$ which admits a sumset filter labelling is called a sumset filter graph.

The following is a necessary and sufficient condition for a sumset labelling $f$ of a given graph $G$ to be a sumset filter labelling of $G$.

Theorem 4.5. [85] An sumset labelling $f$ defined on a given graph $G$ with respect to a non-empty ground set $X$ is a sumset filter labelling of $G$ if and only if the following conditions hold. 
(i) $0 \in X$.

(ii) every subset of $X$ containing 0 is the set-label of some vertex in $G$.

(iii) 0 is an element of the set-label of every vertex in $G$.

If a graph $G$ admits a sumset filter labelling, then it can be observed that $G$ has $2^{|X|-1}$ vertices. Moreover, for a sumset filter graph $G$, only one vertex of $G$ can have a singleton set-label as the only possible singleton set-label in $\mathcal{F}$ is $\{0\}$. Another interesting property for sumset filter graphs is that $f^{+}(E(G)) \subseteq f(V(G))$.

Another interesting structural property of a sumset filter graph has been proved in [85] as follows:

Theorem 4.6. GS21a If a graph $G$ admits a sumset filter labelling, with respect to a non-empty ground set $X$, then $G$ must have at least $2^{|X|-2}$ pendant vertices that are incident on a single vertex of $G$.

If a given graph $G$ admits a sumset filter labelling $f$, then it can be seen that every element of the collection $\mathcal{F}=f(V(G))$ belongs to some finite chain of sets in $\mathcal{F}$ of the form $\{0\}=f\left(v_{1}\right) \subset f\left(v_{2}\right) \subset f\left(v_{3}\right) \subset \ldots \ldots \subset f\left(v_{r}\right)=X$.

The following are some of the immediate observations on the structural characteristics of sumset filter graphs, which are proved in [84].

(i) The existence of a sumset filter labelling is not a hereditary property. That is, a sumset filter labelling of a graph need not induce a sumset filter labelling for all of its subgraphs.

(ii) For $n \geq 3$, no paths $P_{n}$ admits a sumset filter labelling. No cycles admit sumset filter labellings and as a result neither Eulerian graphs nor Hamiltonian graphs admit sumset filter labellings. Neither complete graphs nor complete bipartite graphs admit sumset filter labellings. For $r>2$, complete $r$-partite graphs also do not admit sumset filter labellings.

(iii) Graphs having odd number of vertices never admits a sumset filter labelling.

(iv) Removing any non-leaf edge of a sumset filter graph preserves the sumset filter labelling of that graph. This property is known as monotone property.

\subsection{Graceful and Sequential Sumset Graphs}

Another classification of sumset graphs is according to whether all of the nonempty elements of $\mathcal{P}(X)$, the power set of $X$, appears as the set-labels of of the elements of the graphs concerned. Hence, the notion of graceful sumset labelling of graphs have been introduced in [86] as follows:

Definition 4.4. [86] Let $G$ be a graph and let $X$ be a non-empty set of integers. A sumset indexer $f: V(G) \rightarrow \mathcal{P}_{0}(X)$ is said to be an sumset graceful labelling or a graceful sumset labelling of $G$ if $f^{+}(E(G))=\mathcal{P}(X) \backslash\{\emptyset,\{0\}\}$.

A graph with a graceful sumset labelling, may be called a graceful sumset graph. The structural properties of a graceful sumset graph are very much interesting a 
study in this direction has been done in [86]. Some important properties of them are as follows:

If $f: V(G) \rightarrow \mathcal{P}_{0}(X)$ is a sumset graceful labelling of a given graph $G$, then $\{0\}$ must be the set-label of a vertex in $G$. The vertices of a graceful sumset graph $G$, whose set-labels are the trivial sumsets of any two subsets of $X$, must be adjacent to the vertex $v$ that has the set-label $\{0\}$.

If $x_{1}$ and $x_{2}$ are the minimal and second minimal non-zero elements of the ground set $X$ of a graceful sumset graph $G$, then it can be noted that the vertices of $G$ that have the set-labels $\left\{x_{1}\right\}$ and $\left\{x_{2}\right\}$, must be adjacent to the vertex $v$ that has the set-label $\{0\}$. If $A_{i}$ and $A_{j}$ be two distinct subsets of the ground set $X$ and let $x_{i}$ and $x_{j}$ be the maximal elements of $A_{i}$ and $A_{j}$ respectively, then $A_{i}$ and $A_{j}$ can be the set-labels of two adjacent vertices of a sumset graceful graph $G$ only if $x_{i}+x_{j} \leq x_{n}$, the maximal element of $X$.

Let $f: V(G) \rightarrow \mathcal{P}_{0}(X)$ be a sumset graceful labelling of a given graph $G$ and let $x_{n}$ be the maximal element of $X$. If $A_{i}$ and $A_{j}$ are set-labels of two adjacent vertices, say $v_{i}$ and $v_{j}$, then $A_{i}+A_{j}$ is the set-label of the corresponding edge $v_{i} v_{j}$. Hence, for any $x_{i} \in A_{i}, x_{j} \in A_{j}, x_{i}+x_{j} \leq x_{n}$ and hence, if one of these two sets consists of the maximal element of $X$, then the other set cannot have a non-zero element. Hence, $x_{n}$ is an element of the set-label of a vertex $v$ of $G$ if $v$ is a pendant vertex that is adjacent to the vertex labelled by $\{0\}$. It can also be noted that if $G$ is a graph without pendant vertices, then no vertex of $G$ can have a set-label consisting of the maximal element of the ground set $X$.

Let $f$ be a sumset graceful labelling defined on $G$. Then, $f^{+}(E)=\mathcal{P}(X) \backslash$ $\{\emptyset,\{0\}\}$. Therefore, $|E(G)|=|\mathcal{P}(X)|-2=2^{|X|}-2=2\left(2^{|X|-1}-1\right)$. That is, $G$ has even number of edges. Therefore, the cardinality of the ground set $X$ is $|X|=\log _{2}[|E(G)|+2]$.

Other important structural properties of a graceful sumset graph is explained in the following theorems.

Theorem 4.7. [86] If a graph $G$ admits a sumset graceful labelling $f$ with respect to a finite ground set $X$, then the vertices of $G$, having trivial summands of any subset of $X$ as their set-labels, are the pendant vertices of $G$.

Theorem 4.8. Let $G$ be a sumset graceful graph which admits a sumset graceful labelling $f$ with respect to a finite non-empty set $X$. Then, $G$ must have at least $|X|-1$ pendant vertices.

A graph $G$ which admits a graceful sumset indexer with respect to the set $X$ is said to be a graceful graph-realisation of the set $X$ with respect to the sumset labelling $f$. It is proved in [86] that there exists a non-bipartite graceful graphrealisation $G$ corresponding to any non-empty finite set of integers containing the element 0 .

Let $X$ be a non-empty finite set of integers. Then, a graph $G$ admits a graceful sumset labelling if and only if the following conditions hold (see [86].

(a) $0 \in X$ and $\{0\}$ be a set-label of some vertex, say $v$, of $G$.

(b) the minimum number of pendant vertices in $G$ is the number of subsets of $X$ which are the trivial summands of any subsets of $X$. 
(c) the minimum degree of the vertex $v$ is equal to the number of subsets of $X$ which are not the sumsets of any two subsets of $X$ and not the non-trivial summands of any other subsets of $X$.

(d) the minimum number of pendant vertices that are adjacent to a given vertex of $G$ is the number of subsets of $X$ which are neither the non-trivial sumsets of any two subsets of $X$ nor the non-trivial summands of any subsets of $X$.

Another type of sumset graph is the one with the vertex set-labels and the edge set-labels together form the set $\mathcal{P}_{0}(X)$. Hence, the notion of a sumset sequential labelling of a given graph has been introduced in [87] as follows:

Definition 4.5. A sumset labelling $f$ of $G$ is said to be a sumset sequential labelling or a sequential sumset labelling if the induced function $f^{*}(G)=f(V(G)) \cup$ $f^{+}(E(G))=\mathcal{P}_{0}(X)$, where $f^{*}$ is defined by

$$
f^{*}(x)= \begin{cases}f(x) & \text { if } x \in V(G), \\ f^{+}(x) & \text { if } x \in E(G) .\end{cases}
$$

A graph $G$ which admits a sumset sequential labelling may be called an sequential sumset graph. It can be noted that every sumset graceful labelling of a graph $G$ is also a sumset sequential labelling of $G$.

If $G$ is a sumset sequential graph without isolated vertices and the function $f^{*}$ is an injective function, then one can note that no vertex of $G$ can have a set-label $\{0\}$. Moreover, if one vertex $v$ of $G$ has the set label $\{0\}$, then $v$ should be an isolated vertex of $G$ and no edge of $G$ can also have the set label $\{0\}$.

The following result provides a relation connecting the size and order of a given sumset sequential graph $G$ and the cardinality of its ground set $X$.

Proposition 4.9. [87] Let $G$ be a graph on $n$ vertices and $m$ edges. If $f$ is a sumset sequential labelling of a graph $G$ with respect to a ground set $X$, then $m+n=2^{|X|}-(1+\kappa)$, where $\kappa$ is the number of subsets of $X$ which are the set-labels of both vertices and edges.

Two sets $A$ and $B$ are said to be of same parity if their cardinalities are simultaneously odd or simultaneously even. Then, the following theorem is on the parity of the vertex set and edge set of $G$.

Proposition 4.10. [87] Let $f$ be a sumset sequential labelling of a given graph $G$, with respect to a ground set $X$. Then, if $V(G)$ and $E(G)$ are of same parity, then $\kappa$ is an odd integer and if $V(G)$ and $E(G)$ are of different parity, then $\kappa$ is an even integer, where $\kappa$ is the number of subsets of $X$ which are the set-labels of both vertices and edges.

It is shown in [87] that a graph $G$ that admits a sumset sequential labelling with respect to a non empty ground set $X$ should have at least $\rho$ vertices, where $\rho$ is the number of elements in $\mathcal{P}_{0}(X)$ which are not the sumsets of any two elements of $\mathcal{P}_{0}(X)$. Let $\mathcal{B}$ be the collection of subsets of $X$ which are neither the sumsets of any two subsets of $X$ nor their sumsets are subsets of $X$. If $\mathcal{B}$ is non-empty, then for a sequential sumset graph $G$, it is established in [87] that 
1. $\{0\}$ is the set-label of a vertex in $G$,

2. the minimum number pendant vertices in $G$ is equal to the cardinality of $\mathcal{B}$.

and as result, a sequential sumset graph $G$ should have at least one pendant vertex.

\section{Hypergraphs associated with sumset graphs}

In terms of the set-labels of different sumset graphs, some new types of hypergraphs graphs have been introduced and studied the properties of these discrete structures in [82]. Three new types of hypergraphs associated with the set-labels of the elements of given sumset graphs have been introduced and their structural properties have been studied in [82].

Definition 5.1. [82] Let $X \subset \mathbb{Z}$ and a set-valued function $f: V(G) \rightarrow \mathcal{P}_{0}(X)$ be a sumset labelling of a given graph $G$. Then, the hypergraph $\mathcal{H}(X, \mathcal{L}(V)$ is called the vertex set-label hypergraph (VSL-hypergraph) of $G$ with respect to the sumset labelling $f$ where $\mathcal{L}(V)=\{f(v): v \in V(G)\}$. The VSL-hypergraph of a sumset graph $G$ is denoted by $\mathcal{H}_{G}^{f}$.

In a similar way, the notion of edge set-label hypergraphs of a given sumset graph $G$ has been defined in [82] as follows.

Definition 5.2. [82] Let $X \subset \mathbb{Z}$ and $f: V(G) \rightarrow \mathcal{P}_{0}(X)$ be a sumset labelling of a given graph $G$. Then, a hypergraph $(X, \mathcal{L}(E))$ is called the edge set-label hypergraph (ESL-hypergraph) of $G$ with respect to the sumset labelling $f$, where $\mathcal{L}(E)=\left\{f^{+}(e): e \in E(G)\right\}$. The ESL-hypergraph of a sumset graph $G$ is denoted by $\mathcal{H}_{G}^{f^{+}}$.

Another type of hypergraph associated with a given sumset labelling graph $G$, namely total set-label hypergraph, is defined as follows.

Definition 5.3. [82] Let $X \subset \mathbb{Z}$ and $f: V(G) \rightarrow \mathcal{P}_{0}(X)$ be a sumset labelling of a given graph $G$. Then, a hypergraph $(X, \mathcal{L}(G)$ is called the total set-label hypergraph (TSL-hypergraph) of $G$ with respect to the sumset labelling $f$, where

$$
\mathcal{L}(G)=\left\{\begin{array}{ll}
f(x) & \text { if } x \in V(G) \\
f^{+}(x) & \text { if } x \in E(G) .
\end{array} .\right.
$$

The TSL-hypergraph of a sumset graph $G$ is denoted by $\mathcal{H}_{G}^{*}$.

In the first observation, it is clear that if a sumset graph $G$ has no pendant vertex, then its vertex set-label hypergraph $\mathcal{H}_{G}^{f}$ has some isolated vertices. A hypergraph is said to be a connected hypergraph if its intersection graph is connected. In view of the above facts, a necessary condition for a vertex set-label hypergraph of a sumset graph to be connected is established in [82] as follows:

Proposition 5.1. [82] If the vertex set-label hypergraph of a sumset graph $G$ is a connected hypergraph, then $G$ must have some pendant vertices. 
The converse of Proposition 5.1 is also not true in general. The existence of a connected VSL-hypergraph for a given sumset graph $G$ is established in the following proposition.

Proposition 5.2. [82] Let $G$ be a graph with at least one pendant vertex. Then, there exists a sumset labelling $f$ defined on $G$ such that the corresponding VSLhypergraph $\mathcal{H}_{G}^{f}$ is connected.

Invoking Proposition 5.1 and Proposition 5.2, a necessary and sufficient condition for the VSL-hypergraph $\mathcal{H}_{G}^{f}$ of a given sumset graph $G$ to be a connected hypergraph has been proposed in [82] as given below.

Theorem 5.3. [82] Let $f: V(G) \rightarrow \mathcal{P}_{0}(X)$ be a sumset labelling defined on a given graph $G$, where $X$ is a non-empty set of integers. Then, the VSL-hypergraph $\mathcal{H}_{G}^{f}$ of $G$ is a connected hypergraph if and only if the following conditions hold.

(a) $0 \in X$ and $\{0\}$ is the set-label of a vertex, say $v_{1}$ of $G$

(b) there exists at least one pendant vertex, say $v_{i}$ in $G$ which is adjacent to $v_{1}$ and the set-label of which contains the elements of $X$ which are the trivial summands of any element of $X$.

A hypergraph $\mathcal{H}$ is said to be a uniform hypergraph (or a $k$-uniform hypergraph) if all the hyperedges of $G$ are of the same size (or the size $k$ ). The following results are obvious conditions for the VSL-hypergraphs to be uniform hypergraphs.

Proposition 5.4. [82] Let $f$ be a sumset labelling defined on a graph $G$. Then, the VSL-hypergraph $\mathcal{H}_{G}^{f}$ of $G f$ is a k-uniform hypergraph if and only if $V(G)$ is $k$-uniformly set-indexed.

A hypergraph is said to be a linear hypergraph if any two of its hyperedges have at most one element (vertex) in common. Hence, the conditions required for the hypergraphs associated to a given sumset graph to be linear hypergraphs is provided in the following result.

Theorem 5.5. [82] Let $G$ be a graph with some pendant vertices, which admits a sumset labelling $f$. Then, the VSL-hypergraph $\mathcal{H}_{G}^{f}$ of $G$ is a connected linear hypergraph if and only if the set labels of any two vertices of $G$ contain at most one common element and for every vertex in $G$, there exists at least one vertex in $G$ such that their set-labels have exactly one common element.

It can be noticed that the VSL-hypergraphs associated with the topological sumset graphs, topogenic sumset graphs and certain types of graceful and sequential sumset graphs are obviously connected.

The similar results for edge set-label hypergraphs have also been discussed in [82]. A necessary condition for the ESL-hypergraph $\mathcal{H}_{G}^{f^{+}}$of $G$ to have isolated vertices is as follows.

Proposition 5.6. [82] Let $f: V(G) \rightarrow \mathcal{P}_{0}(X)$ be a sumset labelling of a given graph $G$. If $0 \notin X$, then its ESL-hypergraph $\mathcal{H}_{G}^{f^{+}}$has some isolated vertices. 
The following proposition established a condition for the ESL-hypergraph $\mathcal{H}_{G}^{f^{+}}$ of a sumset graph $G$ to be a connected hypergraph.

Proposition 5.7. [82] Let $G$ be a graph with at least one pendant vertex. Then, there exists a sumset labelling $f$ defined on $G$ such that the ESL-hypergraph $\mathcal{H}_{G}^{f^{+}}$is connected.

In this context also, we can see that the ESL-hypergraphs associated with the topological sumset graphs and topogenic sumset graphs are connected hypergraphs.

In view of the above propositions, a necessary and sufficient condition for an ESL-hypergraph to be a connected hypergraph has been proved in [82] as follows.

Theorem 5.8. [82] Let $G$ be a graph which admits a sumset labelling $f: V(G) \rightarrow$ $\mathcal{P}_{0}(X)$, where $X$ is a non-empty set of integers. Then, the ESL-hypergraph $\mathcal{H}_{G}^{f^{+}}$of $G$ is a connected hypergraph if and only if the following conditions hold.

(a) $0 \in X$ and $\{0\}$ is the set-label of a vertex, say $v_{1}$ of $G$,

(b) there exists at least one pendant vertex, say $v_{i}$, in $G$ which is adjacent to $v_{1}$ and the set-label of which contains the elements of $X$ that are the trivial sumsets of any elements of $X$.

Two hypergraphs are said to isomorphic if their intersection graphs are isomorphic. A necessary condition for the isomorphism between the VSL-hypergraph and the ESL-hypergraph of a sumset labelling graph is provided in [82] as follows.

Theorem 5.9. [82] If the VSL-hypergraph and the ESL-hypergraph of a sumset graph $G$ are isomorphic, then $G$ is a unicyclic graph.

A necessary and sufficient condition for an ESL-hypergraph of a sumset graph $G$ to be a uniform hypergraph has been discussed [82] as given below.

Proposition 5.10. [82] Let $f$ be a sumset labelling defined on a graph $G$. Then, the ESL-hypergraph $\mathcal{H}_{G}^{f^{+}}$of $G$ is a $k$-uniform hypergraph if and only if $f$ is a $k$-uniform sumset labelling defined on $G$.

A necessary and sufficient condition for the ESL-hypergraph $\mathcal{H}_{G}^{f^{+}}$of a sumset labelling graph $G$ to be a connected linear hypergraph is as follows.

Theorem 5.11. [82] Let $G$ be a graph with pendant vertices, which admits a sumset labelling $f$. Then, the ESL-hypergraph $\mathcal{H}_{G}^{f^{+}}$of $G$ is a connected linear hypergraph if and only if

(a) for any two adjacent pairs of vertices $\left(v_{i}, v_{j}\right)$ and $\left(v_{r}, v_{s}\right)$ there exists at most one element in $f\left(v_{i}\right) \times f\left(v_{j}\right)$ that is compatible with an element in $f\left(v_{r}\right) \times f\left(v_{s}\right)$ and vice versa.

(b) for every pair of adjacent vertices $\left(v_{i}, v_{j}\right)$ of $G$, there exist at least one more pair of adjacent vertices $\left(v_{r}, v_{s}\right)$ such that we can find a unique element $(a, b) \in$ $f\left(v_{i}\right) \times f\left(v_{j}\right)$ and a unique element $(c, d) \in f\left(v_{r}\right) \times f\left(v_{s}\right)$ such that $(a, b)$ and $(c, d)$ are compatible. 
The existence of a connected total set-label hypergraph for a given sumset graph is established in the following result.

Proposition 5.12. [82] For any given graph $G$, there exists a sumset labelling $f$ such that the corresponding TSL-hypergraph is connected.

The following theorem established a necessary and sufficient condition for the TSL-hypergraph of a sumset graph $G$ to be a uniform hypergraph.

Theorem 5.13. [82] The TSL-hypergraph of a sumset graph $G$ be a uniform hypergraph if and only if $G$ is 1-uniform.

A necessary condition for a TSL-hypergraph $\mathcal{H}_{G}^{*}$ of a sumset graph $G$ to be a uniform hypergraph is proved in [82] as follows.

Theorem 5.14. If the TSL-hypergraph $\mathcal{H}_{G}^{*}$ of a given sumset graph $G$ is a uniform hypergraph, then $\mathcal{H}_{G}^{*}$ is a disconnected hypergraph.

\section{Sumset Labelled Signed Graphs}

A signed graph (see [94,95]), denoted by $S(G, \sigma)$, is a graph $G(V, E)$ together with a function $\sigma: E(G) \rightarrow\{+,-\}$ that assigns a sign, either + or - , to each ordinary edge in $G$. The function $\sigma$ is called the signature or sign function of $S$, which is defined on all edges except half edges and is required to be positive on free loops. An edge $e$ of a signed graph $S$ is said to be a positive edge if $\sigma(e)=+$ and an edge $\sigma(e)$ of a signed graph $S$ is said to be a negative edge if $\sigma(e)=-$.

A simple cycle (or path) of a signed graph $S$ is said to be balanced if the product of signs of its edges is + . A signed graph is said to be a balanced signed graph if it contains no half edges and all of its simple cycles are balanced. It is to be noted that the number of all negative signed graph is balanced if and only if it is bipartite. Two sets $A$ and $B$ are said to be same parity vertices if the cardinality of both are simultaneously odd or simultaneously even. The sets which do not have same parity are usually called opposite parity sets. In a set-valued graph $G$, two vertices $u$ and $v$ are said to be same parity vertices if their set-labels are same parity sets. Otherwise, they are said to be opposite parity vertices.

\subsection{Sumset Signed Graphs}

The structural characteristics of the signed graphs associated with different types of sumset graphs have been studied in [88-90]. The notion of a sumset labelling of signed graph is introduced in [88] as follows.

Definition 6.1. Let $X \subseteq \mathbb{Z}$ and let $S$ be a signed graph, with corresponding underlying graph $G$ and the signature $\sigma$. An injective function $f: V(S) \rightarrow \mathcal{P}_{0}(X)$ is said to be an sumset labelling of $S$ if $f$ is a sumset labelling of the underlying graph $G$ and the signature of $S$ is defined by $\sigma(u v))=(-1)^{|f(u)+f(v)|}$. A signed graph which admits a sumset labelling is called an sumset signed graph (sumset signed graph) and is denoted by $S_{f}$. 
Definition 6.2. An sumset labelling $f$ of a signed graph $S$ is called a weak sumset labelling or a strong sumset labelling or an arithmetic sumset labelling of $S$, in accordance with the sumset labelling $f$ of the underlying graph $G$ is a weak sumset labelling or a strong sumset labelling or an arithmetic sumset labelling of the corresponding underlying graph $G$.

The following result provides a necessary and sufficient condition for the existence of a balanced signed graph corresponding to a strongly uniform sumset graph.

Theorem 6.1. [88] A strongly $k$-uniform sumset signed graph $S$ is balanced if and only if the underlying graph $G$ is a bipartite graph or $\sqrt{k}$ is an even integer.

The following result provides a solution to this problem of finding the conditions required for a strongly uniform sumset signed graph to be clusterable.

Proposition 6.2. [88] A strongly k-uniform sumset signed graph $S$ is clusterable if and only if the underlying graph $G$ is bipartite and $k$ is an odd integer.

If the underlying graph $G$ of a strong sumset signed graph $S$ is bipartite, then $S$ is balanced if and only if the number of negative edges in $S$ in every cycle of $G$ is even. This is possible only when the number of distinct pairs of adjacent vertices, having odd parity set-labels, in every cycle of $S$ is even. Therefore, we have.

Proposition 6.3. [88] Let $S$ be a strong sumset signed graph with the underlying graph $G$, bipartite. Then, $S$ is clusterable if and only if the number of distinct pairs of adjacent vertices having odd parity set-labels is even.

The following result describes the conditions required for the clusterability of (non-uniform) strong sumset signed graphs whose underlying graph $G$ is a bipartite graph.

Proposition 6.4. [88] The strong sumset signed graph, whose underlying graph $G$ is a bipartite graph, is clusterable if and only if there exist at least two adjacent vertices in $S_{f}$ with odd parity set-labels.

A necessary and sufficient condition for a strong sumset signed graph to be clusterable is described in the following theorem.

Theorem 6.5. [88] A strong sumset signed graph $S$ is clusterable if and only if every odd cycle of the underlying graph $G$ has at least two adjacent vertices with even parity set-label and at least two adjacent vertices with odd parity set-label.

Balance and clusterability of the induced signed graphs of weak sumset graphs are described in the following theorems.

Proposition 6.6. [88] A weakly k-uniform sumset signed graph is always balanced.

The following theorem discussed the clusterability of weakly uniform sumset signed graphs.

Theorem 6.7. [88] A weakly k-uniform sumset signed graph $S$ is clusterable if and only if $k$ is a positive odd integer. 
Balance of a weak sumset signed graph whose underlying graph is a bipartite graph is discussed in the following result.

Proposition 6.8. [88] A weak sumset signed graph $S$, whose underlying graph $G$ is a bipartite graph, is balanced if and only if the number of odd parity non-singleton set-labels in every cycle of $S$ is even.

The following theorem establishes a necessary and sufficient condition for a weak sumset signed graph whose underlying graph is a bipartite graph.

Theorem 6.9. [88] The weak sumset signed graph $S$, whose underlying graph $G$ is a bipartite graph, is clusterable if and only if some non-singleton vertex set-labels of $S$ are of odd parity.

The balance property of weak sumset signed graphs whose underlying graphs are non-bipartite have been discussed the following theorem.

Theorem 6.10. [88] A weak sumset signed graph $S$, whose underlying graph $G$ is a non-bipartite graph, is not balanced.

Corollary 6.11. [88] A weak sumset signed graph $S$, whose underlying graph $G$ is non-bipartite, is not clusterable.

The following theorem for an edge of an arithmetic sumset graph to be a positive edge.

Theorem 6.12. [89] Let $\S$ be a signed graph which admits an arithmetic sumset labelling $f$ of a graph $G$. Then, an edge uv is a positive edge of a sumset signed graph if and only if

(i) the set-labels $f(u)$ and $f(v)$ are of different parity, provided the deterministic ratio of the edge uv is odd.

(ii) the set-label of the end vertex, with minimum common difference, is of even parity, provided the deterministic ratio of the edge uv is even.

The following theorem establishes a necessary and sufficient condition for an arithmetic sumset signed graph to a balanced signed graph.

Theorem 6.13. [89] A sumset signed graph $\S$ is balanced if and only if its underlying graph $G$ is bipartite.

The following theorem described a necessary and sufficient condition for an isoarithmetic sumset signed graph to be balanced.

Theorem 6.14. [88] An isoarithmetic sumset signed graph $S$ is balanced if and only if every cycle in $S$ has even number of distinct pairs of adjacent vertices having the same parity set-labels.

The following result provides the required conditions required for an isoarithmetic sumset signed graph to be clusterable. 
Proposition 6.15. [88] An isoarithmetic sumset signed graph $S$ is clusterable if and only if $S$ contains some disjoint pairs of adjacent vertices having the same parity set-labels.

All set-labels mentioned in this section are arithmetic progressions so that the given signed graph $S$ admits an arithmetic sumset labelling. Invoking the above results, we establish the following theorem for an edges of an arithmetic sumset graph to be a positive edge.

Theorem 6.16. [88] Let $S$ be a signed graph which admits an arithmetic sumset labelling $f$. Then, an edge uv is a positive edge of a sumset labelled signed graph if and only if

(i) the set-labels $f(u)$ and $f(v)$ are of different parity, provided the deterministic ratio of the edge uv is odd.

(ii) the set-label of the end vertex, with minimum common difference, is of even parity, provided the deterministic ratio of the edge uv is even.

The following theorem established a necessary and sufficient condition for an arithmetic sumset signed graph to a balanced signed graph.

Theorem 6.17. [88] An arithmetic sumset signed graph $S$ is balanced if and only if its underlying graph $G$ is bipartite.

A spanned signed subgraph $S^{\prime}$ of a signed graph $S$ is a signed graph which preserves signature and whose underlying graph $H$ is a spanning subgraph of the underlying graph $G$ of $S$. The following result is an obvious and immediate from the corresponding definition of the balanced signed graphs.

Theorem 6.18. [88] Let $S^{\prime}$ be a spanned signed subgraph of a balanced arithmetic sumset signed graph $S$. Then, $S^{\prime}$ is balanced with respect to induced labelling and signature if and only if the following conditions are hold.

(i) the set $E\left(S \backslash S^{\prime}\right)$ contains even number of negative edges in $S$, if the signed graph $S$ is edge disjoint.

(ii) the set $E\left(S \backslash S^{\prime}\right)$ contains odd number of negative edges in $S$ if some of the negative edges are common to two more cycles in $S$.

\subsection{Switching of Sumset Signed Graphs}

The switching of signed graph is the process of changing the sign of the edges in $S$ whose end vertices have different sign. We denote the switched signed graph of a signed graph $S$ by $S^{\prime}$. The following theorem discusses the nature of the switched signed graph of a weak sumset signed graph.

Theorem 6.19. [90] The switched signed graph of a weak sumset signed graph is always an all-negative signed graph. 
Invoking the above theorem, a necessary and sufficient condition for the switched signed graph $S^{\prime}$ of a weak sumset signed graph $S$ is balanced is established in the following result.

Theorem 6.20. [90] The switched signed graph $S^{\prime}$ of a weak sumset signed graph $S$ is balanced if and only if the underlying graph of $S$ (and $S^{\prime}$ ) is a bipartite graph.

The following result discusses the conditions required for the switched signed graph of a strong sumset signed graph to be a homogeneous signed graph.

Theorem 6.21. [90] The switched signed graph $S^{\prime}$ of a strong sumset signed graph $S$ is an all-negative signed graph if and only if at least one end vertex of every edge of $S$ is an odd parity vertex.

From the last paragraph of the above proof, we can observe an immediate result as stated below.

Theorem 6.22. [90] The switched signed graph $S^{\prime}$ of a strong sumset signed graph $S$ is an all-positive signed graph if and only if all vertices in $S$ are even parity vertices.

A necessary and sufficient condition for a signed graph $S$ to admit an arithmetic sumset labelling is found out in [90] as given below.

Theorem 6.23. [90] A signed graph $S$ admits an arithmetic sumset labelling $f$ if and only if for every edge of $S$, the set-labels of its end vertices are arithmetic progressions with common differences $d_{u}$ and $d_{v}$ such that $d_{u} \leq d_{v}$ and its deterministic ratio $\frac{d_{v}}{d_{u}}$ is a positive integer less than or equal to $|f(u)|$.

The following theorem discussed the nature of the switched signed graphs of isoarithmetic sumset signed graphs.

Theorem 6.24. [90] The switched signed graphs of an isoarithmetic sumset signed graph $S$ is an all-negative homogeneous signed graph.

The following theorem discussed a necessary and sufficient condition for the switched signed graphs of isoarithmetic sumset signed graphs to be balanced.

Theorem 6.25. [90] The switched signed graph $S^{\prime}$ of an isoarithmetic sumset signed graph $S$ is balanced if and only if the underlying graph $G$ of $S$ is bipartite.

Next, assume that the deterministic ratio $k$ of the edges in an arithmetic sumset signed graph $S$ is greater than 1 . Then, we have the following theorem.

Theorem 6.26. [90] The switched signed graph $S^{\prime}$ of an arithmetic sumset signed graph $S$ is an all-negative homogeneous signed graph if and only if $S$ contains no edge with even deterministic ratio, whose end vertices with greater common difference is of even parity.

In view of Theorem 6.26, we can establish a necessary and sufficient condition for the switched signed graph of a given arithmetic sumset signed graph to be an all-positive homogeneous signed graph as follows. 
Theorem 6.27. [90] The switched signed graph $S^{\prime}$ of an arithmetic sumset signed graph $S$ is an all-positive signed graph if and only if the following conditions hold.

(i) The deterministic ratio of every edge of $S$ is even; and

(ii) the end vertex with greater common difference of every edge of $S$ is an even parity vertex.

Theorem 6.28. If $S$ is a prime arithmetic sumset signed graph with deterministic ratio $p>2$, then its switched signed graph $S^{\prime}$ is an all-negative signed graph.

Theorem 6.29. The switched signed graph $S^{\prime}$ of a prime arithmetic sumset signed graph $S$, with deterministic ratio $p>2$, is balanced if and only if its underlying graph is a bipartite graph.

\section{Modular Sumset Labelling of Graphs}

Let $n$ be a positive integer. We denote the set of all non-negative integers modulo $n$ by $\mathbb{Z}_{n}$ and its power set by $\mathcal{P}\left(\mathbb{Z}_{n}\right)$. The modular sumset of the two subsets $A$ and $B$ of $\mathbb{Z}_{n}$, denoted by $A+B$, is the set defined by $A+B=\{x: a+b \equiv x(\bmod n), a \in$ $A, b \in B\}$. Through out our discussion, $A+B$ is the sumset of $A$ and $B$. It can also be noted that $A, B \subseteq \mathbb{Z}_{n} \Longrightarrow A+B \subseteq \mathbb{Z}_{n}$.

Then, using the concepts of modular sumsets of sets and analogous to the definition of sumset labellings of graphs, the notion of modular sumset labelling of a graph has been introduced in [47] as follows:

Definition 7.1. [47] A function $f: V(G) \rightarrow \mathcal{P}\left(\mathbb{Z}_{n}\right)$, whose induced function $f^{+}(u v): E(G) \rightarrow \mathcal{P}\left(\mathbb{Z}_{n}\right)$ is defined by $f^{+}(u v)=f(u)+f(v)$, is said to be a modular sumset labelling if $f$ is injective.

It is proved in [47] that every graph admits a modular sumset labelling. A graph $G$ which admits a modular sumset labelling is called an modular sumset graph. In View of the corresponding results on the bounds for the cardinality of sumsets of two sets, the cardinality of the set-label of a modular sumset graph $G$ is given in the following theorem.

Theorem 7.1. [4'] Let $f: V(G) \rightarrow \mathcal{P}\left(\mathbb{Z}_{n}\right)$ be a modular sumset labelling of a given graph $G$. Then, for any edge uv $\in E(G)$, we have $|f(u)|+|f(v)|-1 \leq\left|f^{+}(u v)\right|=$ $|f(u)+f(v)| \leq|f(u)||f(v)| \leq n$.

Analogous to the notions of sumset labelled graphs, the following terms and definitions have been defined for modular sumset graphs.

(a) The cardinality of the set-label of an element of $G$ is said to be the set-labelling number of that element.

(b) If all the vertices of a graph $G$ have the same set-labelling number, say $l$, then we say that $V(G)$ is l-uniformly set-labelled. 
(c) A modular sumset labelling of $G$ is said to be a $k$-uniform modular sumset labelling if the set-labelling number of all edges of $G$ have the same set-labelling number $k$.

(d) The elements of $G$ having the set-labelling number 1 are called the monoindexed elements of $G$.

(e) The smallest value of $n$ such that $f: V(G) \rightarrow \mathcal{P}\left(\mathbb{Z}_{n}\right)$ is a modular sumset labelling of a given graph $G$ is called the modular sumset number of $G$. The modular sumset number of a graph $G$ is denoted by $\eta(G)$.

An edge can be mono-indexed if and only if its end vertices are mono-indexed. The set-labelling number of an edge of a given graph $G$ is equal to the set-labelling number of both of its end vertices if and only if the edge and its end vertices are mono-index.

One can note that the modular sumset number of a graph $G$ is at least $1+\log _{2} m$ (see [47].

The following result establishes the condition for a sumset to have the same cardinality of one or both of its summands.

Proposition 7.2. [47] Let $A$ and $B$ be two non-empty subsets of $\mathbb{Z}_{n}$. Then, $|A+B|=|A|($ or $|A+B|=|B|)$ if and only if either $|A|=|B|=\mathbb{Z}_{n}$ or $|B|=1$ (or $|A|=1)$. More over, $|A+B|=|A|=|B|$ if and only if $|A|=|B|=\mathbb{Z}_{n}$ or $|A|=|B|=\mathbb{Z}_{n}$

Hence, analogous to the corresponding notion of sumset labelled graphs (see $[34,60])$, we have the following definition.

Definition 7.2. [47] A modular sumset labelling $f$ of a graph $G$ is said to be a weak modular sumset labelling of $G$ if the set-labelling number of every edge of $G$ is equal to the set-labelling number of at least one of its end vertices.

A graph $G$ which admits a weak modular sumset labelling is called a weak modular sumset graph. It is to be noted that for a weak modular sumset graph, no two adjacent vertices can have non-singleton set-labels. As in the case of

Hence, analogous to the corresponding of weak sumset labelled graphs the following results are valid for weak modular sumset graphs.

Theorem 7.3. [47] A graph $G$ admits a weak modular sumset labelling if and only if $G$ is bipartite or contains mono-indexed edges.

Theorem 7.4. A graph $G$ admits a weakly uniform modular sumset labelling if and only if $G$ is bipartite.

The notion of the weak modular sumset number of a given graph is introduced in [47] as follows.

Definition 7.3. The weak modular sumset number of a graph $G$ is defined to be the minimum value of $n$ such that a modular sumset labelling $f: V(G) \rightarrow \mathcal{P}\left(\mathbb{Z}_{n}\right)$ is a weak modular sumset labelling of $G$. 
The following theorem determines the weak sumset number of an arbitrary graph $G$ in terms of its covering and independence numbers.

Theorem 7.5. Let $G$ be a modular sumset graph and $\alpha$ and $\beta$ be its covering number and independence number respectively. Then, the weak modular sumset number of $G$ is $\max \{\alpha, r\}$, where $r$ is the smallest positive integer such that $2^{r}-r-1 \geq \beta$.

The weak modular sumset number of many fundamental graph classes has been determined in the paper [88].

The minimum cardinality of the ground set $X$ when the given graph $G$ admits a weakly uniform modular sumset labelling the following result.

The minimum cardinality of the ground set when the given graph $G$ admits a weakly uniform modular sumset labelling arouses much interest in this occasion. Hence, we have the following result.

Theorem 7.6. Let $G$ be a graph with covering number $\alpha$ and independence number $\beta$ and let $G$ admits a weakly $k$-uniform modular sumset labelling, where $k<\alpha$ being a positive integer. Then, the minimum cardinality of the ground set $\mathbb{Z}_{n}$ is $\max \{\alpha, r\}$, where $r$ is the smallest positive integer such that $\left(\begin{array}{l}r \\ k\end{array}\right) \geq \beta$.

Analogous to the corresponding notions on strong sumset labelled graphs, the following notion has been introduced in [47].

Definition 7.4. [47] Let $f: V(G) \rightarrow \mathcal{P}\left(\mathbb{Z}_{n}\right)$ be a modular sumset labelling defined on a given graph $G$. Then, $f$ is said to be a strong modular sumset labelling if for the associated function $f^{+}: E(G) \rightarrow \mathcal{P}\left(\mathbb{Z}_{n}\right),\left|f^{+}(u v)\right|=|f(u)||f(v)| \forall u v \in E(G)$. A graph which admits a strong modular sumset labelling is called a strong modular sumset graph.

Then, a necessary and sufficient condition for a graph to admit a strong modular sumset labelling has been proved in [47] as given below.

Theorem 7.7. [47] A modular sumset labelling $f: V(G) \rightarrow \mathcal{P}\left(\mathbb{Z}_{n}\right)$ of a given graph $G$ is a strong modular sumset labelling of $G$ if and only if $D_{f(u)} \cap D_{f(v)}=\emptyset, \forall u v \in$ $E(G)$, where $|f(u)||f(v)| \leq n$.

Analogous to the weak modular sumset number of a graph $G$, we can define the strong modular sumset number of $G$ as the minimum cardinality required for the ground set $\mathbb{Z}_{n}$ so that $G$ admits a strong modular sumset labelling. The choice of ground set $\mathbb{Z}_{n}$ is very important in this context because $n$ should be sufficiently large so that the vertices of the given graph can be labelled in such a way that the difference sets of these set-labels of all adjacent vertices must be pairwise disjoint.

Analogous to the corresponding results on strong sumset labelled graphs and strongly uniform sumset labelled graphs proved in [60], the following results are valid for strong modular sumset graphs also.

Theorem 7.8. [47] For a positive integer $k \leq n$, a modular sumset labelling $f: V(G) \rightarrow \mathcal{P}\left(\mathbb{Z}_{n}\right)$ of a given connected graph $G$ is a a strongly $k$-uniform modular sumset labelling of $G$ if and only if either $k$ is a perfect square or $G$ is bipartite. 
Theorem 7.9. [47] For a positive non-square integer $k \leq n$, a modular sumset labelling $f: V(G) \rightarrow \mathcal{P}\left(\mathbb{Z}_{n}\right)$ of an arbitrary graph $G$ is a a strongly $k$-uniform modular sumset labelling of $G$ if and only if either $G$ is bipartite or a disjoint union of bipartite components.

For a positive integer $k \leq n$, the maximum number of components in a strongly $k$-uniform modular sumset graph is as follows.

Proposition 7.10. [47] Let $f$ be a strongly $k$-uniform modular sumset labelling of a graph $G$ with respect to the ground set $\mathbb{Z}_{n}$. Then, the maximum number of components in $G$ is the number of distinct pairs of divisors $r$ and $s$ of $k$ such that $r s=k$.

It can be observed that a strongly $k$-uniform modular sumset graph can have a non-bipartite component if and only if $k$ is a perfect square. More over, a strongly $k$-uniform modular sumset graph $G$ can have at most one non-bipartite component.

A modular sumset labelling $f: V(G) \rightarrow \mathcal{P}\left(\mathbb{Z}_{n}\right)$ of a given graph $G$ is a maximal modular sumset labelling of $G$ if the set-label of every edge of $G$ is the ground set $\mathbb{Z}_{n}$ itself. The conditions required for a graph to admit a maximal modular sumset labelling has been found out in [47].

Proposition 7.11. [47] The modular sumset labelling $f: V(G) \rightarrow \mathcal{P}\left(\mathbb{Z}_{n}\right)$ of a given graph $G$ is a maximal modular sumset labelling of $G$ if and only if for every pair of adjacent vertices $u$ and $v$ of $G$ some or all of the following conditions hold.

(i) $|f(u)|+|f(v)| \geq n$ if $D_{f(u)} \cap D_{f(v)} \neq \emptyset$. The strict inequality hold when $D_{f(u)}$ and $D_{f(v)}$ are arithmetic progressions containing the same elements.

(ii) $|f(u)||f(v)| \geq n$ if $D_{f(u)} \cap D_{f(v)}=\emptyset$.

A necessary and sufficient condition for a strong modular sumset labelling of a graph $G$ to be a maximal modular sumset labelling of $G$.

Theorem 7.12. [47] Let $f$ be a strong sumset-labelling of a given graph $G$. Then, $f$ is a maximal sumset-labelling of $G$ if and only if $n$ is a perfect square or $G$ is bipartite or a disjoint union of bipartite components.

\section{Scope for Further Studies}

More properties and characteristics of different types of sumset labellings, both uniform and non-uniform, are yet to be investigated. Many problems regarding the admissibility of sumset labelling by various graph classes, graph operations and graph products are still open. The studies on sumset graphs whose set-labels are well known sequences are also promising for further studies. Some of the problems in this area identified are the following.

Problem 1. Characterise the sumset graphs, whose vertex set-labels are arithmetic progressions, but the edge set-labels are not arithmetic progressions. 
Problem 2. Characterise the arithmetic sumset graphs, the deterministic ratios of whose edges are prime numbers.

A sumset labelling $f$ of a graph $G$ is said to be a bitopological sumset labelling of $G$ if both the collections $f(V) \cup\{\emptyset\}$ and $f^{+}(E) \cup\{\emptyset,\{0\}\}$ are topologies of the ground set $X$.

Problem 3. Verify the existence of graphical realisation of topological sumset labellings corresponding to different standard topologies.

Problem 4. Verify the existence of a certain types of sumset labellings for a given graph $G$ such that the collections of its vertex set-labels and edge set-labels form different algebraic structures such as semigroups, $\sigma$-algebra etc. of the ground set $X$ and determine the structural properties of the graphs which admit these types of sumset labellings.

Analogous to the different types of sumset graphs, the investigation can be extended to the field of modular sumset graphs also

All these facts highlight a wide scope for further investigations in this area.

\section{References}

[1] Abhishek K., (2007). New directions in the theory of set-valuations of graphs, Ph. D Thesis, Kannur University, Kannur, India.

[2] Abhishek K., (2013). Set-valued graphs-II, J. Fuzzy Set Valued Anal., 2013, 1-16, DOI: 10.5899/2013/jfsva-00149.

[3] Abhishek K., (2015). A note on set-indexed graphs, J. Discrete Math. Sci. Cryptography, 18(1-2), 31-40, DOI: 10.1080/09720529.2013.867637.

[4] Abhishek K., (2015). Set-valued graphs: A survey, J. Discrete Math. Sci. Cryptography, 18(1-2), 55-80, DOI: 10.1080/09720529.2014.894306.

[5] Acharya B.D.,(1983). Set-valuations and their applications, MRI Lecture Notes in Applied Mathematics, No.2, The Mehta Research Institute of Mathematics and Mathematical Physics, Allahabad.

[6] Acharya B.D., (2001). Set-indexers of a graph and set-graceful graphs, Bull. Allahabad Math. Soc., 16: 1-23.

[7] Acharya B.D., (2012). Set-valuations of signed digraphs, J. Combin. Inform. System Sci., 37(2-4): 145-167.

[8] Acharya B.D. and Germina K.A., (2013). Set-valuations of graphs and their applications: A survey, Ann. Pure Appl. Math., 4(1), 8-42.

[9] Acharya B.D., Germina K.A., Princy K.L. and Rao S.B., (2008). On setvaluations of graphs, in labelling of Discrete Structures and Applications, (Eds.: Acharya B.D., S. Arumugam and A. Rosa), Narosa Pub. House, New Delhi. 
[10] Acharya B.D., Germina K.A. and Paul V., (2010). Linear hypergraph setindexers of a graph, Int. Math. Forum, 5(68): 3359-3370.

[11] Akiyama J., Avis D. and Chav́tal V. and Era H., (1981). Balancing signed graphs, Discrete Appl. Math., 3(4): 227-233., DOI: 10.1016/0166218X(81)90001-9.

[12] Anandavally T.M.K., (2013). A characterisation of 2-uniform IASI graphs, Int. J. of Contemp. Math. Sci., 8(10), 459-462.

[13] Apostol T.M., (1989). Introduction to analytic number theory, SpringerVerlag, New York.

[14] Berge C., (2001). Theory of graphs, Dover Pub.,

[15] Berge C., (1989). Hypergraphs: Combinatorics of finite sets, North-Holland, Amsterdam.

[16] Berge C., (1979). Graphs and hypergraphs, North-Holland, Amsterdam.

[17] Bollabás B., (1998). Modern graph theory, Springer International Edition.

[18] Bondy J.A. and Murty U.S.R., (2008). Graph theory, Springer.

[19] Brandstädt A., Le V.B. and Spinrad J.P., (1999). Graph classes: A survey, SIAM, Philadelphia.

[20] Burton D.M., (2007). Elementary number theory, Tata McGraw-Hill Inc., New Delhi.

[21] Chartrand G. and Zhang P., (2005). Introduction to graph theory, McGrawHill Inc.

[22] Chartrand G. and Lesniak L., (1996). Graphs and digraphs, CRC Press.

[23] Chithra K.P., Sudev N.K. and Germina K.A., (2014). The sparing number of the Cartesian product of certain graphs, Commun. Math. Appl., 5(1): 23-30.

[24] Chithra K.P., Sudev N.K. and Germina K.A., (2015). On the sparing number of the edge-corona of graphs, Int. J. Comput. Applications, 118(1): 1-5., DOI: $10.5120 / 20706-3025$.

[25] Chithra K.P., Sudev N.K. and Germina K.A., (2014). A study on the sparing number of the corona of certain graphs, Res. Review. Discrete Math. Structure, 1(2): 5-15.

[26] Clark W.E., (2002). Elementary number theory, Department of Mathematics, University of South Florida, USA.

[27] Clarke J. and Holton D.A., (1991). A first look at graph theory, World Scientific Pub., Singapore. 
[28] Deo N., (1974). Graph theory with application to engineering and computer science, Prentice Hall of India Pvt. Ltd., Delhi.

[29] Diestel R., (2010). Graph theory, Springer-Verlag, New York.

[30] Dugundji J., (1966). Topology, Allyn and Bacon, Boston.

[31] Gallian J.A., (2018). A dynamic survey of graph labelling, Electron. J. Combin., (DS-6).

[32] Germina K.A., (2011). Set-valuations of a graph and applications, Final Technical Report, DST Grant-In-Aid Project No.SR/S4/277/05, The Dept. of Science and Technology (DST), Govt. of India.

[33] Germina K.A. and Anandavally T.M.K., (2012). Integer additive set-indexers of a graph: Sum square graphs, J. Combin. Inform. System Sci., 37(2-4):, 345-358.

[34] Germina K.A. and Sudev N.K., (2013). On weakly uniform integer additive set-indexers of graphs, Int. Math. Forum, 8(37): 1827-1834., DOI: 10.12988/imf.2013.310188.

[35] Golomb S.W., (1972). How to number a graph, in Graph Theory and Computing, (Ed.: R C Read), Academic Press: 13-22.

[36] Gross J. and Yellen J., (1999). Graph theory and its applications, CRC Press.

[37] Hammack R., Imrich W. and Clavzar S., (2011). Handbook of product graphs, CRC Press.

[38] Harary F., (1969). Graph theory, Narosa Pub. House, New Delhi.

[39] Harary F., (1953). On the notion of balance of a signed graph, Michigan Math. J., 2(2): 143-146.

[40] Harary F. and Palmer E.M., (1973). Graphical enumeration, Academic Press Inc.,

[41] Joshi K.D., (2003). Applied discrete structures, New Age International, New Delhi.

[42] Joshi K.D., (1983). Introduction to General Topology, New Age International, New Delhi.

[43] Krishnamoorthy V., (1966). On the number of topologies of finite sets, Amer. Math. Monthly, 73(2): 154-157.

[44] McKee T.A. and McMorris F.R., (1999). Topics in intersection graph theory, SIAM, Philadelphia.

[45] Munkers J.R., (2000). Topology, Prentice Hall of India, Delhi. 
[46] Naduvath S., and Augustine G., (2018). An introduction of sumset valued graphs, Lambert Academic Publ., Beau Bassin, Mauritius.

[47] Naduvath S., (2017). A study on modular sumset labelling of graphs, Discrete Math., Algorithm. Appl., 9(1): 1-16, DOI: 10.1142/S1793830917500392.

[48] Nathanson M.B. (1996). Additive number theory: Inverse problems and geometry of sumsets, Springer, New York.

[49] Ore O., Theory of Graphs, (1962). American Math. Soc. Colloquium Pub., XXXV III.

[50] Paul V., and Germina K.A., (2011). On uniform linear hypergraph setindexers of graphs, Int. J. Contemp. Math. Sci., 6(18): 861-868.

[51] Paul V., (2012). Labeling and set-indexing hypergraphs of a graph and related areas, Ph.D. Thesis, Kannur Univ., Kannur, India.

[52] Princy K.L., (2007). Some studies on set-valuations of graph-embedding and NP-completeness, Ph. D Thesis, Kannur Univ., Kannur, India.

[53] Rao S.B. and Germina K.A., (2011). Graph labelings and complexity problems: A review, in Graph Theory Research Directions (Eds: P. Panigrahi and S B Rao), Narosa Pub. House, New Delhi.

[54] Rosa A., (1967). On certain valuation of the vertices of a graph, in Theory of Graphs, Gordon and Breach, New York.

[55] Roberts F.S., (1978). Graph theory and its practical applications to problems of society, SIAM, Philadelphia.

[56] Rousa I.K., (2008). Sumsets and structures, Alfréd Rényi Institute of Mathematics, Budapest.

[57] Singh G.S., (1998). A note on labelings of graphs, Graphs and Combin., 14, 201-207.

[58] Stoll R.R., (1979). Set theory and Logic, Dover pub.

[59] Sudev N.K., (2015). Set-valuations of discrete structures and their applications, PhD Thesis, Kannur University, Kannur, India.

[60] Sudev N.K. and Germina K.A., (2014). On integer additive set-indexers of graphs, Int. J. Math. Sci. Eng. Appl., 8(II): 11-22.

[61] Sudev N.K. and Germina K.A., (2014). A characterization of weak integer additive set-indexers of graphs, J. Fuzzy Set Valued Anal., 2014: 1-7., DOI:10.5899/2014/jfsva-00189.

[62] Sudev N.K. and Germina K.A., (2014). Weak integer additive set-indexers of graph operations, Global J. Math. Sci. Theory. Practical, 6(1): 25-36. 
[63] Sudev N.K. and Germina K.A., (2014). A study of semi-arithmetic integer additive set-indexers of graphs, Int. J. Math. Sci. Eng. Appl., 8(III): 157-165.

[64] Sudev N.K. and Germina K.A., (2014). On the sparing number of certain graph structures, Annals Pure Appl. Math., 6(2): 140-149.

[65] Sudev N.K. and Germina K.A., (2014). Associated graphs of certain arithmetic IASI graphs, Int. J. Math. Soft Comput., 4(2): 71-80.

[66] Sudev N.K. and Germina K.A., (2014). A note on the sparing number of graphs, Adv. Appl. Discrete Math., 14(1): 51-65.

[67] Sudev N.K. and Germina K.A., (2014). Further studies on the sparing number of graphs, TechS Vidya e-J. Res., 2: 25-36.

[68] Sudev N.K. and Germina K.A., (2015). Weak integer additive set indexes of certain graph classes, J. Discrete Math. Sci. Cryptography, 18(2-3): 117-128, DOI:10.1080/09720529.2014.962866.

[69] Sudev N.K. and Germina K.A., (2014). Weak integer additive set-indexers of certain graph products, J. Inform. Math. Sci., 6(1): 35-43.

[70] Sudev N.K., Germina K.A. and Chithra K.P., (2014). Weak set-labelling number of certain integer additive set-labelled graphs, Int. J. Comput. Appl., 114(2): 1-6., DOI: 10.5120/19947-1772.

[71] Sudev N.K., Germina K.A., (2015). Some new results on weak integer additive set-labelling of graphs, Int. J. Comput. Appl., 128(5): 1-5., DOI: $10.5120 /$ ijca2015906514.

[72] Sudev N.K. and Germina K.A., (2014). A Characterisation of strong integer additive set indexers of graphs, Commun. Math. Appl., 5(3): 101-110.

[73] Sudev N.K. and Germina K.A., (2015). Some new results on strong integer additive set indexers of graphs, Discrete Math Algorithm. Appl., 7(1): 1-11., DOI: $10.1142 /$ S1793830914500657.

[74] Sudev N.K. and Germina K.A., (2015). A note on the sparing number on the sieve graphs of certain graphs, Appl Math. E-Notes, 15: 29-37.

[75] Sudev N.K. and Germina K.A., (2015). A study on the nourishing number of graphs and graph power, Math., 3: 29-39; DOI:10.3390/math3010029.

[76] Sudev N.K. and Germina K.A., (2018). Arithmetic integer additive setindexers of certain graph operations, J. Inform. Math. Sci., 10(1-2): 321-332, DOI: $10.26713 /$ jims.v10i1+\&+2.617.

[77] Sudev N.K. and Germina K.A., (2015).A study on topological integer additive set-labelling of graphs, Electron. J. Graph Theory Appl., 3(1): 70-84, DOI: 10.5614/ejgta.2015.3.1.8. 
[78] Sudev N.K. and Germina K.A., (2015). On certain arithmetic integer additive set-indexers of graphs, Discrete Math. Algorithm. Appl.,7(3): 1-15., DOI:10.1142/S1793830915500251.

[79] Sudev N.K. and Germina K.A., (2017). A study on prime arithmetic integer additive set-indexers of graphs, Proyecciones J. Math., 36(2):195-208, DOI: 10.4067/S0716-09172017000200195.

[80] Sudev N.K. and Germina K.A., (2014). A study on semi-arithmetic setindexers of graphs, Int. J. Math. Sci. Eng. Appl., 8(III): 157-165.

[81] Sudev N.K. and Germina K.A., (2015). Some new results on weak integer additive set-labelled graphs, Int. J. Computer Appl., 128(1): 1-5, DOI:10.5120/ijca2015906514.

[82] Sudev N.K. and Germina K.A., (2015). On the hypergraphs associated with certain integer additive set-labelled graphs, J. Adv. Res. Appl. Math.,7(4): 23-33., DOI: 10.5373/jaram.2287.021015.

[83] Sudev N.K. and Germina K.A., (2015). On integer additive set-sequential graphs, Int. J. Math. Combin., 2015(3): 125-133.

[84] Sudev N.K. and Germina K.A., (2015).A study on topogenic integer additive set-labelled graphs, J. Adv. Res. Pure Math., 7(3): 15-22., DOI: $10.5373 / \mathrm{jarpm} .2230 .121314$.

[85] Sudev N.K., Chithra K.P., Germina K.A., (2015). On integer additive setfilter graphs, J. Abst. Comput. Math., 3: 8-15.

[86] Sudev N.K. and Germina K.A., (2019). A study on integer additive setgraceful graphs, Southeast Asian Bull. Math., to appear.

[87] Sudev N.K. and Germina K.A., (2015). On integer additive set-sequential graphs, Int. J. Math. Combin., 2015(3): 125-133.

[88] Sudev N.K. and Germina K.A., (2015). Integer additive set-valuations of signed graphs, Carpathian Math. Publ., 7(2): 236-246.

[89] Sudev N.K., Ashraf P.K. and Germina K.A., (2019). Integer additive setvaluations of signed graphs, TWMS J. Appl. Engg. Math., to appear.

[90] Sudev N.K. and Germina K.A., (2015). Switched signed graphs of integer additive set-valued signed graphs, Discrete Math. Algorithm. Appl., 9(4): 110, DOI: $10.1142 /$ S1793830917500434.

[91] Thomas B.K., (2009). Advanced studies on labelling of graphs and hypergraphs and related areas, Ph.D. Thesis, Kannur University, Kannur, India.

[92] Voloshin V.I., (2009). Introduction to graph and hypergraph theory, Nova Science Pub., New York. 
[93] West D.B., (2001). Introduction to graph theory, Pearson Education Inc.

[94] Zaslavsky T., (1982). Signed graphs, Discrete Appl. Math., 4(1): 47-74., DOI: 10.1016/0166-218X(82)90033-6.

[95] Zaslavsky T., (2012). Signed graphs and geometry, J. Combin. Inform. System Sci., 37(2-4): 95-143.

[96] Zaslavsky T., (1981). Characterizations of signed graphs, J. Graph Theory, 5(4):, 401-406., DOI: 10.1002/jgt.3190050409.

[97] Zaslavsky T., (2010). Balance and clustering in signed graphs, C R Rao Adv. Instt. of Mathematics Statistics and Computer Science, Hyderabad, India. 